OPEN ACCESS

Edited by:

Meng Xie,

Brookhaven National Laboratory,

United States

Reviewed by:

Hiroshi Noguchi,

Nihon Pharmaceutical University,

Japan

Yali Sun,

Oak Ridge National Laboratory, United States

Laura E. Bartley,

Washington State University,

United States

*Correspondence:

Ronald R. Sederoff

Ron_Sederoff@ncsu.edu

Jack P. Wang

JPWang@ncsu.edu

Vincent L. Chiang

VChiang@ncsu.edu

${ }^{t}$ These authors have contributed equally to this work

Specialty section:

This article was submitted to

Plant Metabolism

and Chemodiversity,

a section of the journal

Frontiers in Plant Science

Received: 20 June 2021 Accepted: 13 September 2021

Published: 06 October 2021

Citation:

Lin C-Y, Sun Y, Song J, Chen H-C, Shi R, Yang C, Liu J, Tunlaya-Anukit S, Liu B, Loziuk PL,

Williams CM, Muddiman DC

Lin Y-CJ, Sederoff RR, Wang JP and

Chiang VL (2021) Enzyme Complexes of Ptr4CL and PtrHCT Modulate

Co-enzyme A Ligation

of Hydroxycinnamic Acids for Monolignol Biosynthesis

in Populus trichocarpa.

Front. Plant Sci. 12:727932.

doi: 10.3389/fpls.2021.727932

\section{Enzyme Complexes of Ptr4CL and PtrHCT Modulate Co-enzyme A Ligation of Hydroxycinnamic Acids for Monolignol Biosynthesis in Populus trichocarpa}

\author{
Chien-Yuan Lin ${ }^{1,2 t}$, Yi Sun ${ }^{3 t}$, Jina Song ${ }^{41}$, Hsi-Chuan Chen ${ }^{1}$, Rui Shi ${ }^{1}$, Chenmin Yang ${ }^{1}$, \\ Jie Liu', Sermsawat Tunlaya-Anukit', Baoguang Liu'3,5, Philip L. Loziuk6, \\ Cranos M. Williams ${ }^{4}$, David C. Muddiman', Ying-Chung Jimmy Lin ${ }^{1,3}$, \\ Ronald R. Sederoff1*, Jack P. Wang ${ }^{1,3 *}$ and Vincent L. Chiang ${ }^{1,3 *}$ \\ ${ }^{1}$ Forest Biotechnology Group, Department of Forestry and Environmental Resources, North Carolina State University, \\ Raleigh, NC, United States, ${ }^{2}$ Joint BioEnergy Institute, Lawrence Berkeley National Laboratory, Berkeley, CA, United States, \\ ${ }^{3}$ State Key Laboratory of Tree Genetics and Breeding, Northeast Forestry University, Harbin, China, ${ }^{4}$ Department \\ of Electrical and Computer Engineering, North Carolina State University, Raleigh, NC, United States, ${ }^{5}$ Department \\ of Forestry, Beihua University, Jilin, China, ${ }^{6}$ W.M. Keck FTMS Laboratory, Department of Chemistry, North Carolina State \\ University, Raleigh, NC, United States
}

Co-enzyme A (CoA) ligation of hydroxycinnamic acids by 4-coumaric acid:CoA ligase $(4 \mathrm{CL})$ is a critical step in the biosynthesis of monolignols. Perturbation of $4 \mathrm{CL}$ activity significantly impacts the lignin content of diverse plant species. In Populus trichocarpa, two well-studied xylem-specific Ptr4CLs (Ptr4CL3 and Ptr4CL5) catalyze the CoA ligation of 4-coumaric acid to 4-coumaroyl-CoA and caffeic acid to caffeoylCoA. Subsequently, two 4-hydroxycinnamoyl-CoA:shikimic acid hydroxycinnamoyl transferases (PtrHCT1 and PtrHCT6) mediate the conversion of 4-coumaroyl-CoA to caffeoyl-CoA. Here, we show that the CoA ligation of 4-coumaric and caffeic acids is modulated by Ptr4CL/PtrHCT protein complexes. Downregulation of PtrHCTs reduced Ptr4CL activities in the stem-differentiating xylem (SDX) of transgenic $P$. trichocarpa. The Ptr4CL/PtrHCT interactions were then validated in vivo using biomolecular fluorescence complementation (BiFC) and protein pull-down assays in P. trichocarpa SDX extracts. Enzyme activity assays using recombinant proteins of Ptr4CL and PtrHCT showed elevated CoA ligation activity for Ptr4CL when supplemented with PtrHCT. Numerical analyses based on an evolutionary computation of the CoA ligation activity estimated the stoichiometry of the protein complex to consist of one Ptr4CL and two PtrHCTs, which was experimentally confirmed by chemical cross-linking using SDX plant protein extracts and recombinant proteins. Based on these results, we propose that Ptr4CL/PtrHCT complexes modulate the metabolic flux of CoA ligation for monolignol biosynthesis during wood formation in $P$. trichocarpa.

Keywords: protein interaction, monolignol biosynthesis, wood formation, Populus trichocarpa, BiFC, metabolic flux 


\section{INTRODUCTION}

Lignin was first described in 1839 as an "incrusting material of wood" by Payen (1839). Since then, there have been many diverse efforts to resolve the function, structure, formation, and biodegradation of this intriguing constituent of the plant secondary cell wall (SCW) (Sarkanen and Ludwig, 1971; Gross, 1979; Higuchi, 1985, 1990; Boerjan et al., 2003; Weng and Chapple, 2010; Ralph et al., 2019). Lignin is one of three major components in the SCW of vascular plants, along with cellulose and hemicelluloses (Sarkar et al., 2009; Albersheim et al., 2010). In the SCW, lignin interweaves with cellulose and hemicelluloses to form lignin-carbohydrate complexes (LCCs) through covalent linkages, such as (phenyl) glycosidic, acetal, ester, and ether bonds (Jung, 1989; Jin et al., 2006; Du et al., 2013, 2014; Tarasov et al., 2018). Depending on the plant species and biomass origin, lignin content can vary from $18-35 \%$ in woody plants and $7-30 \%$ in herbaceous plants (Sun and Cheng, 2002; Rowell et al., 2005; Pauly and Keegstra, 2008; Gupta et al., 2014).

Lignin is a heterogeneous phenolic polymer and the second most abundant biopolymer on earth, accounting for $\sim 30 \%$ of the organic carbon in the terrestrial biosphere (Boerjan et al., 2003). Lignin is synthesized through oxidative radical coupling (Ralph et al., 2004) and polymerized from three canonical monolignol precursors, 4-coumaryl alcohol, coniferyl alcohol, and sinapyl alcohol, which form the three major subunits of lignin, referred to as 4-hydroxyphenyl (H), guaiacyl (G), and syringyl (S) subunits, respectively (Higuchi, 1997). The composition of lignin is highly malleable (Sederoff et al., 1999; Ralph, 2010; Vanholme et al., 2012; Mottiar et al., 2016) and can vary greatly in the proportion of traditional subunits, in the types of linkages, and in the incorporation of non-canonical monomers into the lignin polymer. The incorporation of dihydroconiferyl alcohol (Ralph et al., 1997), caffeyl alcohol (Chen et al., 2012), ferulate (Wilkerson et al., 2014), tricin (Lan et al., 2015), hydroxystilbene (Carlos del Río et al., 2017), benzoate (Kim et al., 2020), and hydroxycinnamic amides (del Río et al., 2020) in lignin have been documented.

The deposition of lignin is crucial to support the plant body by providing mechanical rigidity, facilitating water transport due to its hydrophobicity, and preventing natural biomass degradation by its highly irregular chemical structure and its resistance to biotic and abiotic stresses (Hu et al., 1999; Tzin and Galili, 2010; Miedes et al., 2014; Bomble et al., 2017). Lignin also confers recalcitrance to lignocellulosic feedstocks for bioenergy and industrial applications, adversely impacting biomass utilization for biofuel and pulp/paper production (Himmel, 2009). Lignin content, composition, and biomass density are significant contributors to biomass recalcitrance (Campbell and Sederoff, 1996; Novaes et al., 2010).

In angiosperms, the lignin is predominantly polymerized from $\mathrm{S}$ and $\mathrm{G}$ monolignols, while in most gymnosperms, lignin is

Abbreviations: AC, affinity chromatography; BiFC, biomolecular fluorescence complementation; DSP, dithiobis (succinimidyl propionate); IP, immunoprecipitation; LCC, lignin-carbohydrate complexes; PC-IDMS, protein cleavage isotope dilution mass spectrometry; RNAi, RNA interference; RPM, reads per million; SCW, secondary cell wall; SDX, stem-differentiating xylem; TAP, tandem affinity purification (TAP); Y2H, yeast two-hybrid. mainly formed from $\mathrm{G}$ monolignols. The relative abundance of $\mathrm{H}$ monolignols is usually below $1 \%$ in the angiosperms, but it can be higher in monocot grasses ( $5 \%)$ (Barrière et al., 2007; Vanholme et al., 2010) or more elevated in compression wood of gymnosperms (Yeh et al., 2006; Nanayakkara et al., 2009). Moreover, within the same plant species, the deposition of different types of monolignols can also vary. For example, in woody dicots, $G$ subunits are enriched in vessels, and S subunits are enriched in fibers (Wang et al., 2018). The spatiotemporal deposition of monolignols within individual cell types could be modulated in an enzyme-specific or cell typespecific manner during monolignol biosynthesis, which remains to be investigated further (Guo et al., 2010; Zhao et al., 2010; Barros and Dixon, 2020).

Monolignol biosynthesis, originates from the shikimate pathway (Brown and Neish, 1955; Vogt, 2010), is often depicted as a biosynthetic grid rather than a linear pathway (Figure 1). The monolignol biosynthetic pathway begins with the conversion of phenylalanine through sequential enzymatic reactions, starting with deamination, followed by hydroxylation, coenzyme A (CoA)-ligation, transesterification, methylation, reduction, and oxidation (Whetten and Sederoff, 1995; Higuchi, 2003; Raes et al., 2003; Hamberger et al., 2007; Liu, 2012; Lu et al., 2013; Shen et al., 2013; Lin et al., 2016; Deng and Lu, 2017). In Populus trichocarpa, 23 xylem-specific enzymes within 11 protein families were proposed to be involved in monolignol biosynthesis, which includes ammonia lyase [e.g., phenylalanine ammonia-lyase (PAL)], ligase [e.g., 4-coumaric acid:CoA ligase (4CL)], acyltransferase [e.g., 4-hydroxycinnamoylCoA:shikimic acid hydroxycinnamoyl transferase (HCT)], hydrolase [e.g., caffeoyl shikimate esterase (CSE)], reductase [e.g., cinnamoyl CoA reductase (CCR)], alcohol dehydrogenase [e.g., cinnamyl alcohol dehydrogenase (CAD)], methyltransferase [e.g., caffeoyl-CoA O-methyltransferase (CCoAOMT), 5hydroxyconiferaldehyde $O$-methyltransferase $\quad(\mathrm{COMT})]$, and cytochrome P450 monooxygenases [e.g., cinnamate 4hydroxylase $(\mathrm{C} 4 \mathrm{H})$, p-coumaroyl shikimate 3 '-hydroxylase $\left(\mathrm{C}^{\prime} \mathrm{H}\right)$, and coniferaldehyde 5-hydroxylase (CAld5H)] (Shi et al., 2010; Saleme et al., 2017; Wang et al., 2018). Recently, in Brachypodium distachyon and Arabidopsis thaliana, a nonmembrane bound cytosolic ascorbate peroxidase that catalyzes the direct 3-hydroxylation of 4-coumarate to caffeate in lignin biosynthesis was identified as a coumarate 3-hydroxylase $(\mathrm{C} 3 \mathrm{H})$, which should be distinguished from the activity of $\mathrm{C}^{\prime} \mathrm{H}$ (Barros et al., 2019). Highly coordinated mechanisms among the monolignol biosynthetic enzymes have been revealed in several model plants and described quantitatively using mathematical modeling (Lee and Voit, 2010; Lee et al., 2012; Wang et al., 2014; Faraji et al., 2015, 2018). Many strategies have been successfully deployed to reduce the recalcitrance of the cell walls to improve biomass utilization by identifying natural variants (MacKay et al., 1997; Studer et al., 2011; Chanoca et al., 2019) or by genetic manipulation of the relative abundance of the monolignol biosynthetic enzymes (Eckardt, 2002; Baucher et al., 2003; Vanholme et al., 2008; Weng et al., 2008; Poovaiah et al., 2014; Peña-Castro et al., 2017; Wang et al., 2018). Conversion of lignin to valued-added bioproducts has also been proposed by lignin valorization (Ragauskas et al., 2014; Upton and Kasko, 2016; 


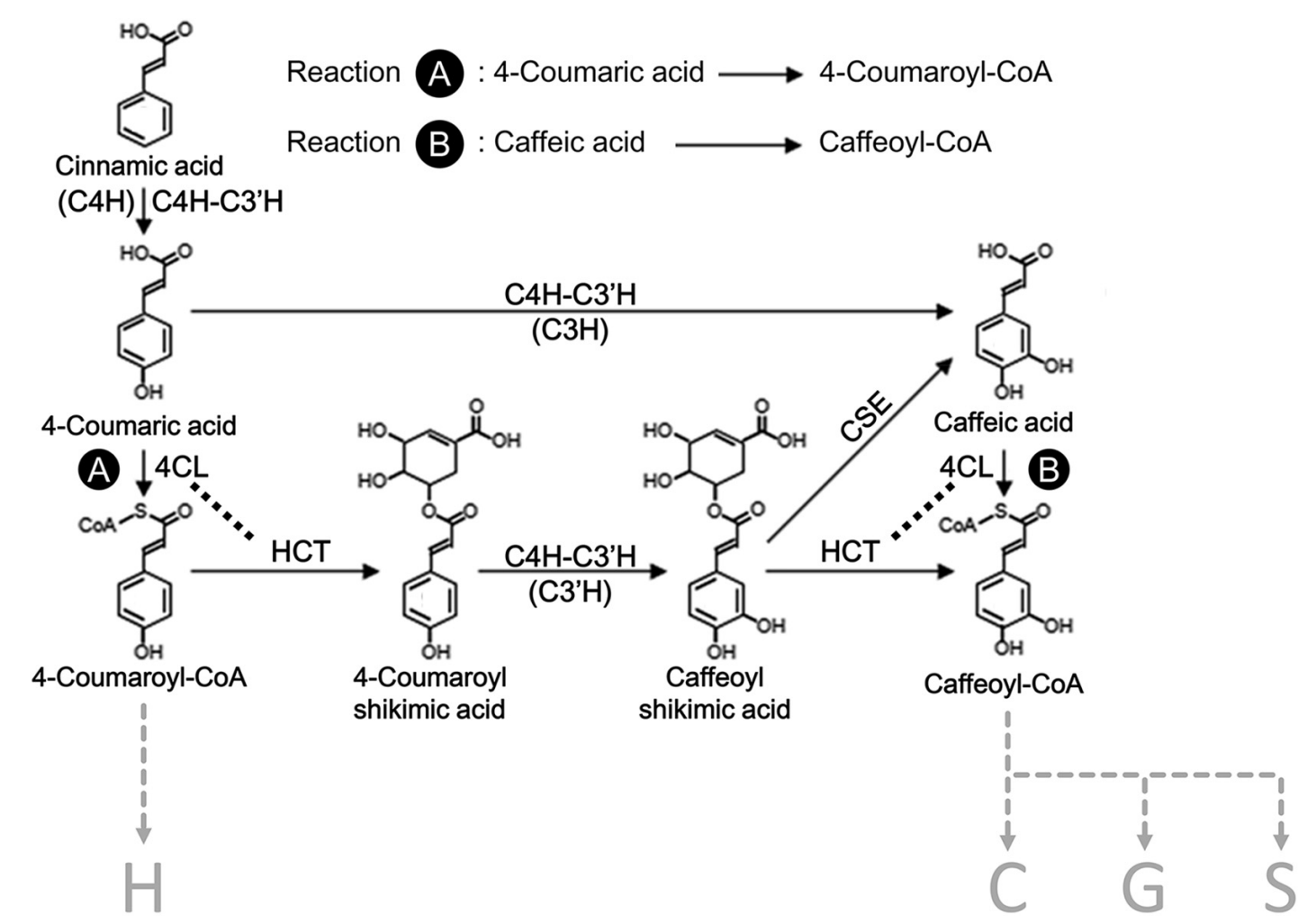

FIGURE 1 | A section of the proposed monolignol biosynthetic pathway in Populus spp. The H, C, G, and S refer to the type of subunits in lignin, 4-hydroxyphenyl, catechyl, guaiacyl, and syringyl, respectively. Reaction A indicates $4 \mathrm{CL}$ activity for the formation of 4-coumaroyl-CoA from 4-coumaric acid, and reaction B indicates $4 \mathrm{CL}$ activity for the formation of caffeoyl-CoA from caffeic acid. $\mathrm{C} 4 \mathrm{H}$, cinnamate 4-hydroxylase; $\mathrm{C} 3 \mathrm{H}, p$-coumarate 3-hydroxylase; $\mathrm{C}^{\prime} \mathrm{H}, p$-coumaroyl shikimate 3'-hydroxylase; 4CL, 4-coumarate CoA ligase; HCT, 4-hydroxycinnamoyl-CoA:shikimic acid hydroxycinnamoyl transferase; CSE, caffeoyl shikimate esterase. Enzymes in parenthesis indicate the distinguished reactions for the specific hydroxylation of monolignols.

Gillet et al., 2017) and lignin manipulation (Welker et al., 2015; Lin and Eudes, 2020).

Although metabolic grids describe pathways as twodimensional processes, most intracellular enzymes operate as aggregates with tight or loose association within biosynthetic sequences (Nester et al., 1967; Ginsburg and Stadtman, 1970; Winkel, 2009; Sweetlove and Fernie, 2013; Schmitt and An, 2017). These protein aggregates can be multienzyme complexes, which are sometimes conceived to be a set of functionally related and physically associated proteins with a highly organized structure (Ginsburg and Stadtman, 1970). Different proteinprotein interactions among the biosynthetic enzymes within the multienzyme complexes can possess physiologically significant regulatory roles, such as compartmentation, metabolite channeling, or catalytic facilitation (Gaertner et al., 1970; Masters, 1977; Stafford, 1981; Luckner, 1984; Hrazdina and Wagner, 1985a; Hrazdina and Jensen, 1992; Winkel, 2004; Williams et al., 2011). Several multienzyme complexes have been described for the biosyntheses of tryptophan (Miles, 2001), shikimate (Giles et al., 1967), polyamines (Panicot et al., 2002), camalexin (Mucha et al., 2019), and cyanogenic glucosides (Laursen et al., 2016). The "supramolecular complexes of sequential metabolic enzymes and cellular structural elements" have been defined as metabolons, which may be anchored on the endoplasmic reticulum (ER) by membrane-bound P450 monooxygenases (P450s) (Srere, 1985; Ovádi and Sreret, 1999; Weid et al., 2004; Jorgensen et al., 2005; Laursen et al., 2015; Obata, 2019).

Since 1974, multienzyme complexes were proposed for aromatic metabolism, including C6-C3 phenolic compounds, flavonoids, and monolignol biosynthesis (Stafford, 1974a,b; Winkel-Shirley, 1999, 2001). The metabolism of flavonoids and monolignols, involving PAL, $\mathrm{C} 4 \mathrm{H}$, and chalcone synthase (CHS), was proposed to be colocalized on the cytoplasmic face of the ER from Hippeastrum (Wagner and Hrazdina, 1984), and the involvement of P450s in phenylpropanoid metabolism had been suggested as membrane anchors (Ralston and Yu, 2006). A loose membrane-associated enzyme complex, including PAL, CHS, uridine diphosphate glucose (UDPG):flavonoid glucosyl transferase (UFGT), was revealed in buckwheat (Fagopyrum esculentum) to facilitate flavonoid biosynthesis through putative interactions with membrane anchors, such as $\mathrm{C} 4 \mathrm{H}$ or flavonoid 3'-hydroxylase $\left(\mathrm{F}^{\prime} \mathrm{H}\right)$ (Hrazdina and Wagner, 1985b; Hrazdina et al., 1987; Hrazdina, 1992). Evidence supports a flavonoid metabolon in A. thaliana (Burbulis and Winkel-Shirley, 1999), in which $\mathrm{CHS}$ and chalcone isomerase (CHI) interact with flavanone 3-hydroxylase $(\mathrm{F} 3 \mathrm{H})$ or dihydroflavonol 4-reductase (DFR) were confirmed by yeast two-hybrid (Y2H) interactions, 
affinity chromatography (AC) or co-immunoprecipitation (IP) assays (Burbulis and Winkel-Shirley, 1999). The participation of $\mathrm{F}^{\prime} \mathrm{H}$ in the flavonoid metabolon had also been shown in Arabidopsis and rice. Using an Arabidopsis $t t 7$ mutant that lacks the $\mathrm{F}^{\prime} \mathrm{H}$, the colocalization of $\mathrm{CHS}$ and $\mathrm{CHI}$ was disrupted (Winkel-Shirley, 2001; Winkel, 2004), while the association of $\mathrm{F}^{\prime} \mathrm{H}$ with $\mathrm{CHS} 1$ has been demonstrated in rice using Y2H (Shih et al., 2008). Differential physical interactions among flavonoid enzymes with flavone synthase II (FNSII) for metabolon formation in flavone/anthocyanin biosynthesis had been elucidated in the plant Order Lamiales (mints, which includes snapdragons and torenia) using splitubiquitin $\mathrm{Y} 2 \mathrm{H}$ and biomolecular fluorescence complementation (BiFC) (Fujino et al., 2018). An isoflavonoid metabolon was also confirmed in soybean with the involvement of soluble enzymes [CHS, CHI, chalcone reductase (CHR)], and twin membrane-bound anchors [C4H and isoflavones synthase (IFS)] (Dastmalchi et al., 2016; Waki et al., 2016; Mameda et al., 2018). A bottleneck for isoflavone production was previously identified by the competition for flavanone between IFS and flavonoid biosynthesis (Liu et al., 2002).

In contrast to the more extensive understanding of multienzyme complexes involved in flavonoid biosynthesis, the identification of protein-protein interaction among monolignol biosynthetic enzymes has been long-postulated but not unambiguously established (Wagner and Hrazdina, 1984; Hrazdina and Wagner, 1985b; Winkel-Shirley, 1999; Biała and Jasiński, 2018). A membrane-bound enzyme complex was first reported at the entry point of the phenylpropanoid pathway using microsomal membrane fractions from potato tubers (Czichi and Kindl, 1975). The PAL-C4H association can use cinnamic acid formed by the PAL reaction as a more effective substrate than cinnamic acid added exogenously (Czichi and Kindl, 1977), while the disruption of the PAL-C4H interaction during the preparation of microsomes led to increased conversion of exogenous cinnamic acid using buckwheat seedlings (Hrazdina and Wagner, 1985b). Direct interactions for PAL-C4H were demonstrated in Nicotiana tabacum, by in vivo isotopic labeling and fluorescence energy resonance transfer (FRET) microscopy, to strengthen the evidence for metabolic channeling for the entry point of monolignol biosynthesis (Rasmussen and Dixon, 1999; Achnine et al., 2004).

Besides the PAL-C4H interaction, protein-protein interactions among the monolignol biosynthetic enzymes have been discovered around the early steps of the phenylpropanoid pathway through the advancements in plant biotechnology. In Arabidopsis, CYP73A5 $(\mathrm{AtC} 4 \mathrm{H})$ and CYP98A3 $\left(\mathrm{AtC}^{\prime} \mathrm{H}\right)$ are colocalized on the ER membrane where they form homo- and heteromers, which is confirmed by fluorescence microscopy and tandem affinity purification (TAP) (Bassard et al., 2012). Two membrane steroid-binding proteins (AtMSBP1 and AtMSBP2) were recently identified to function as a scaffold to interact with all of the three monolignol P450 enzymes (AtC3 ${ }^{\prime} \mathrm{H}, \mathrm{AtC} 4 \mathrm{H}$, and AtF5H) by $\mathrm{Y} 2 \mathrm{H}, \mathrm{BiFC}, \mathrm{LC}-\mathrm{MS}$, and IP assays (Gou et al., 2018). In Populus trichocarpa, a multienzyme membrane-bound protein complex among monolignol biosynthetic enzymes of $\mathrm{PtrC}^{\prime} \mathrm{H} 3 / \mathrm{PtrC} 4 \mathrm{H} 1 / \mathrm{PtrC} 4 \mathrm{H} 2$ has been identified to catalyze both 4- and 3-hydroxylation of cinnamic acid derivatives in monolignol biosynthesis, which drastically increased enzyme metabolic efficiency (Chen et al., 2011). A heterotetrameric protein complex of Ptr4CL3-Ptr4CL5 was identified with novel enzymatic specificity, and the metabolic regulation of CoA ligation by the complex has been quantitatively described by predictive mathematical modeling (Chen et al., 2014).

Following the proposed metabolic sequence for the early steps of monolignol biosynthesis, HCT is expected to be the downstream partner of 4CL (Figure 1). Based on advanced fluorescence microscopy, a closer association of the ER membrane with At4CL1 and AtHCT was proposed for Arabidopsis (Bassard et al., 2012). However, the physiological function of the protein-protein interaction between 4CL and HCT has not been further characterized. To extend the understanding of the proposed lignin metabolon, we investigated the protein-protein interaction between Ptr4CL and PtrHCT in $P$. trichocarpa with a focus on the CoA-ligation function of Ptr4CL, which is crucial for the regulation of phenylpropanoid metabolic flux. First, we generated PtrHCT downregulated transgenic $P$. trichocarpa using RNA interference (RNAi). When PtrHCT1 or PtrHCT6 was downregulated in the PtrHCT RNAi transgenic plants, we observed a PtrHCT-dependent reduction in the CoA-ligation activities of Ptr4CLs, indicating a Ptr4CLPtrHCT interaction. Second, we confirmed the Ptr4CL-PtrHCT protein-protein interaction using BiFC and pull-down assays. The Ptr4CL-PtrHCT enzyme complex was confirmed in vitro using purified recombinant enzymes. Using enzyme assays of mixed recombinant proteins, a numerical model was constructed to describe the behavior of Ptr4CLs in the Ptr4CL-PtrHCT complex and to predict the stoichiometry of the protein complex based on evolutionary computation (Chen et al., 2014). The mathematical model estimated the stoichiometry of the Ptr4CLPtrHCT complex to be one subunit of Ptr4CL and two subunits of PtrHCT. Finally, the Ptr4CL-PtrHCT enzyme complex was validated by chemical cross-linking in wood forming tissues of $P$. trichocarpa, supporting the stoichiometry and molecular weight of the predicted Ptr4CL-PtrHCT complex.

\section{RESULTS}

\section{Co-enzyme A Ligation Activity of Ptr4CL Is Reduced When PtrHCT Is Downregulated}

The CoA ligation of hydroxycinnamic acids by $4 \mathrm{CL}$ creates an activated state of the acids in phenylpropanoid metabolism for monolignol biosynthesis. To investigate whether HCT interacts with $4 \mathrm{CL}$ and the biological significance of an interaction, we measured the $4 \mathrm{CL}$ activity when the expression of HCT was downregulated by RNA interference (RNAi). Previously, in P. trichocarpa, PtrHCT1 and PtrHCT6 were identified as two xylem-specific and xylem-abundant members of the PtrHCT family involved in monolignol biosynthesis (Shi et al., 2010). Three independent transgenic lines with specific downregulation of PtrHCT1 or PtrHCT6 by RNAi transgenesis were obtained 
(Supplementary Figure 1), which were used to evaluate how perturbations of PtrHCT may affect the activity, transcript level, and protein abundance of Ptr4CL.

First, crude protein extracts of stem-differentiating xylem (SDX) were used to measure the CoA ligation activities of 4CL toward 4-coumaric acid (Reaction A) and caffeic acid (Reaction B). Compared to wildtype (WT), 4CL activity was reduced by $30-47 \%$ in reaction $A$ and by $29-43 \%$ in reaction $\mathrm{B}$ when PtrHCT1 was downregulated; while in the case of PtrHCT6 downregulation, the 4CL activity was also reduced by $31-65 \%$ in reaction $\mathrm{A}$ and by 23 $70 \%$ in reaction $\mathrm{B}$ (Figure 2A). We then confirmed that the reduction in $4 \mathrm{CL}$ activity was not a result of reduced $4 \mathrm{CL}$ expression. Full transcriptome RNA-seq showed no significant difference in the transcript abundance of Ptr4CL3 or Ptr4CL5 between the HCT transgenics and WT P. trichocarpa (Figure 2B) and the absolute protein abundances of Ptr4CL3 and Ptr4CL5 were also consistent in the SDX of HCT transgenics and WT using protein cleavage isotope dilution mass spectrometry (PC-IDMS) (Supplementary Figure 2A). The specific downregulation of either PtrHCT1 or PtrHCT6 in the corresponding transgenic lines (Supplementary Figure 2B) suggests that the catalytic facilitation of Ptr4CL reactions by PtrHCT is specific, therefore PtrHCT may play a regulatory role in the CoA-ligation activities.

\section{Protein-Protein Interactions Between Ptr4CL and PtrHCT Revealed by BiFC}

The presence of protein-protein interactions between Ptr4CLs and PtrHCTs was investigated using reciprocal BiFC. By fusing the Ptr4CLs or PtrHCTs to the N-terminal fragment of YFP $\left(\mathrm{YFP}^{\mathrm{N}}\right)$ or the C-terminal fragment of YFP $\left(\mathrm{YFP}^{\mathrm{C}}\right)$, we constructed eight BiFC vectors (Ptr4CL3-YFP ${ }^{\mathrm{N}}$, Ptr4CL3-YFPC, Ptr4CL5-YFP ${ }^{\mathrm{N}}$, Ptr4CL5-YFPC, PtrHCT1-YFPN, PtrHCT1$\mathrm{YFP}^{\mathrm{C}}$, PtrHCT6-YFP ${ }^{\mathrm{N}}$, and PtrHCT6-YFPC ) to evaluate their interactions systematically. Pair-wise combinations of $\mathrm{BiFC}$ vectors were co-transfected into $P$. trichocarpa SDX protoplasts. Positive YFP fluorescence was recorded as indication of Ptr4CL and PtrHCT interactions.

Bright YFP fluorescence signals were detected in P. trichocarpa SDX protoplasts when Ptr4CL3-YFP ${ }^{\mathrm{N}}$ was co-transfected with either PtrHCT1-YFP ${ }^{\mathrm{C}}$ or PtrHCT6-YFPC (Figures 3A,C) and, as well as, when Ptr4CL5-YFP ${ }^{\mathrm{N}}$ was co-transfected with either PtrHCT1-YFPC or PtrHCT6-YFPC (Figures 3E,G). Furthermore, the fluorescence signals of YFP were observed for reciprocal pair-wise combinations (Figures 3B,D,F,H). A negative control was included to validate the results of the BiFC assays by co-transfecting a $\beta$-glucuronidase (Gus)fused YFP fragment individually with the eight BiFC vectors. No fluorescence signal was detected when Gus-YFPC was co-transfected with Ptr4CL3-YFP ${ }^{\mathrm{N}}$, Ptr4CL5-YFP ${ }^{\mathrm{N}}$, PtrHCT1$\mathrm{YFP}^{\mathrm{N}}$, and PtrHCT6-YFP ${ }^{\mathrm{N}}$ (Figures 3I-L), confirming the specificity of the interactions. The $\mathrm{BiFC}$ results revealed that Ptr4CLs (Ptr4CL3 and Ptr4CL5) might directly or indirectly interact with PtrHCTs (PtrHCT1 and PtrHCT6) in P. trichocarpa.

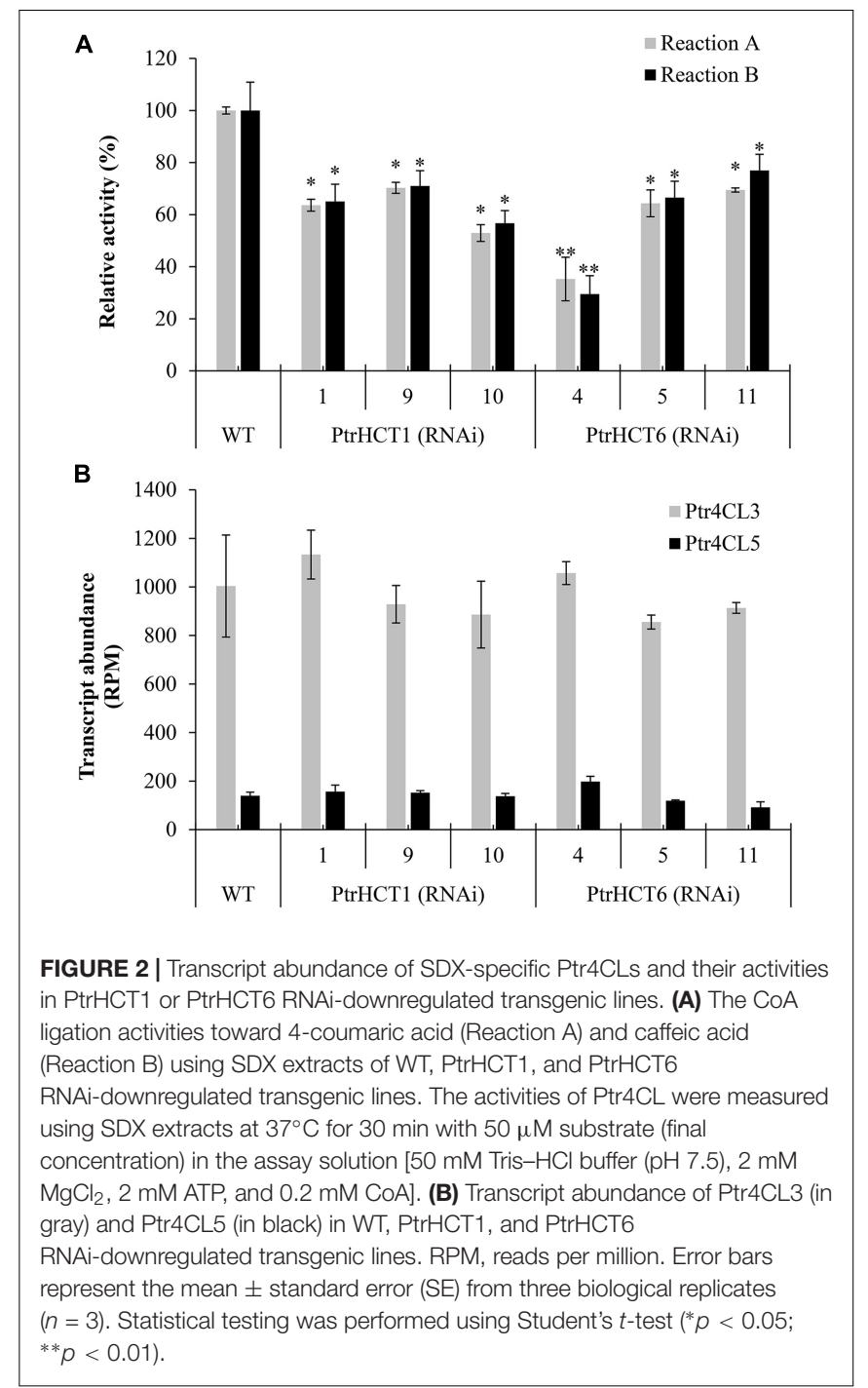

\section{Protein Pull-Down Assays Support the Formation of Ptr4CL-PtrHCT Complexes}

We performed pull-down assays in SDX protein extracts of $P$. trichocarpa to verify the interactions between Ptr4CLs and PtrHCTs (Figure 4). Polyclonal antibodies for Ptr4CLs and PtrHCTs were produced and validated for their protein specificity in SDX extracts and purified recombinant proteins using western blotting. All four antibodies (anti-Ptr4CL3, antiPtr4CL5, anti-PtrHCT1, and anti-PtrHCT6) could specifically bind to their target proteins with minimal non-specific binding to other members of their protein families, confirming the suitability of these antibodies for use in pull-down assays (Supplementary Figure 3).

If stable interactions exist between PtrHCTs and Ptr4CLs, the protein complexes could be separated from SDX crude extracts by affinity purification of one of the interacting proteins. The other interacting proteins could then be identified by western blotting using the protein-specific antibodies. Recombinant proteins of Ptr4CL3, Ptr4CL5, PtrHCT1, and PtrHCT6 fused with 

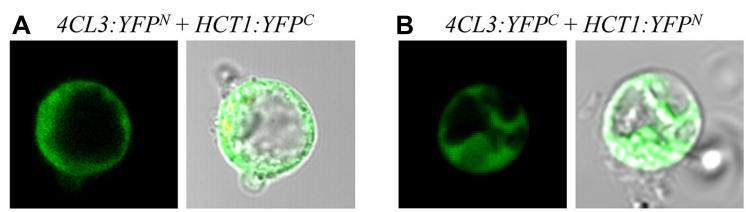

C $4 C L 3: Y F P^{N}+H C T 6: Y F P^{C}$

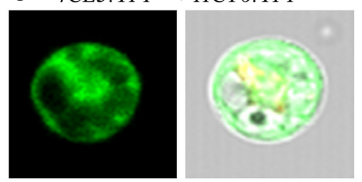

E $\quad 4 C L 5: Y F P^{N}+H C T 1: Y F P^{C}$
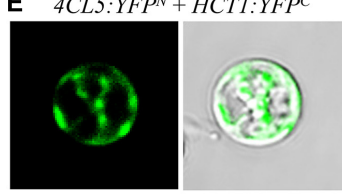

G $4 C L 5: Y F P^{N}+H C T 6: Y F P^{C}$

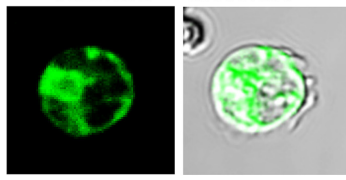

I $H C T 1: Y F P^{N}+$ Gus:YFPC

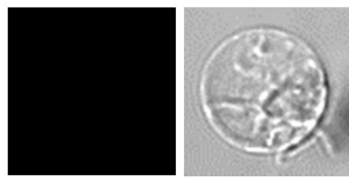

K $4 C L 3: Y F P^{N}+$ Gus:YFPC
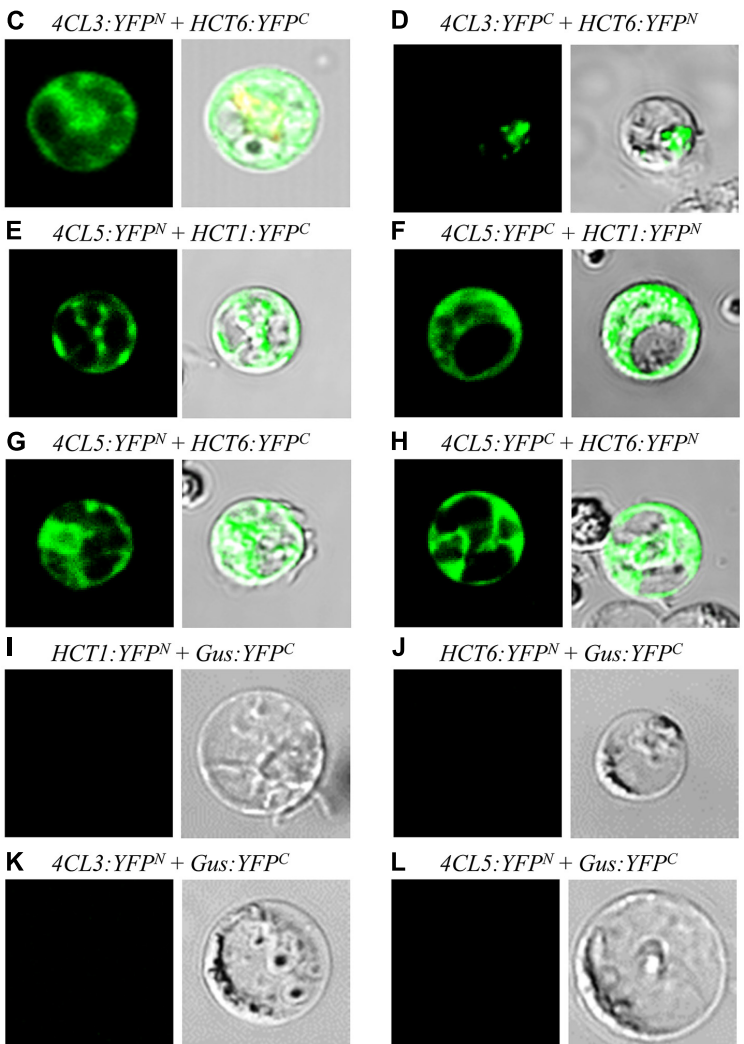

H $4 C L 5: Y F P^{C}+H C T 6: Y F P^{N}$

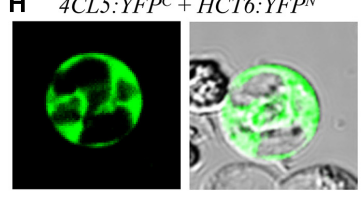

J HCT6:YFP + Gus:YFPC

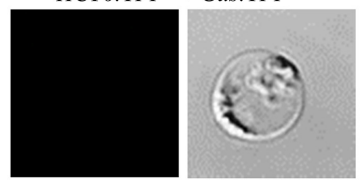

$\mathbf{L} \quad 4 C L 5: Y F P^{N}+$ Gus:YFPC

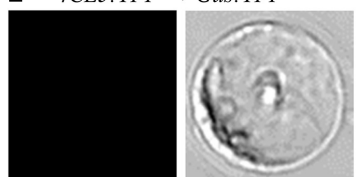

FIGURE 3 | Protein-protein interactions between Ptr4CLs and PtrHCTs were detected by reciprocal bimolecular fluorescence complementation (BiFC).

(A) Ptr4CL3-YFPN was cotransformed with PtrHCT1-YFPC

(C) Ptr4CL3-YFPN was cotransformed with PtrHCT6-YFPC

(E) Ptr4CL5-YFPN was cotransformed with PtrHCT1-YFPC.

(G) Ptr4CL5-YFPN was cotransformed with PtrHCT6-YFPC. (B,D,F,H)

Reciprocal BiFC assays of (A,C,E,G). (I-L) Negative controls of BiFC assays.

The Gus-YFPC were cotransformed with PtrHCT1-YFPN , PtrHCT6-YFPN (E),

or Ptr4CL3-YFPN , Ptr4CL5-YFPN (F), as the negative controls.

C- terminal six-histidine-tags $(6 \times$ His $)$ were individually mixed with SDX crude extracts and then purified by immobilized nickelaffinity chromatography to isolate their interacting proteins. Gus$6 \times$ His mixed with SDX crude extracts was included as a negative control. When Ptr4CL3-6 × His, Ptr4CL5-6 × His, PtrHCT1$6 \times$ His, and PtrHCT6-6 $\times$ His were affinity purified from SDX, Ptr4CL3 was detected in all the pull-down samples using protein-specific antibodies (Figure 4A). Consistently, Ptr4CL5 was also detected as an interacting protein in all the pulldown samples (Figure 4B). None of the target proteins were detected when Gus- $6 \times$ His was pulled down from SDX crude extracts, confirming the specificity of the assays for identifying interacting proteins (Figures $4 \mathbf{A}, \mathbf{B}$ ). As positive controls, all the His-tag-fused recombinant proteins were able to be detected

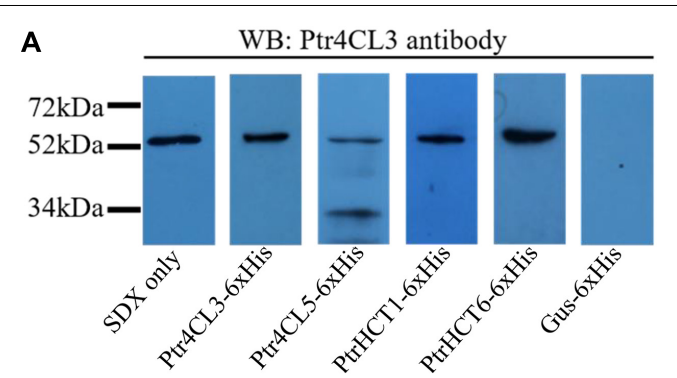

B

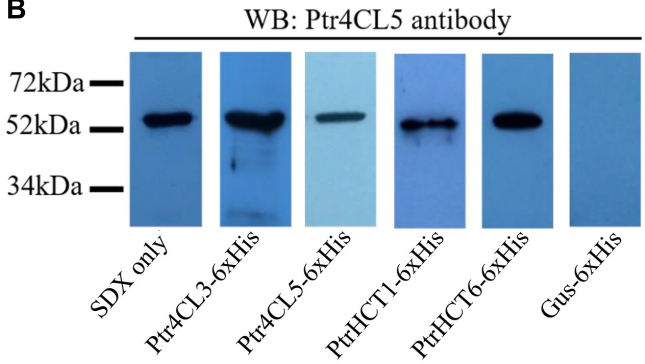

C

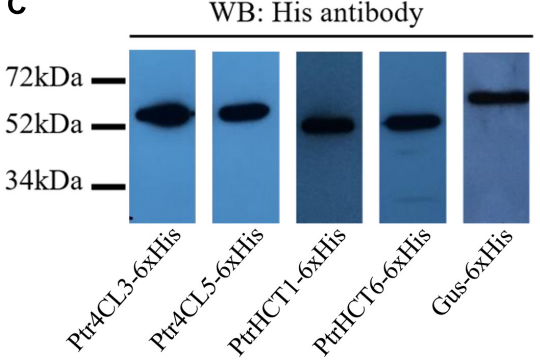

FIGURE 4 | Protein-protein interactions between Ptr4CLs and PtrHCTs were detected on western blots after pull-downs using SDX total protein extracts. SDX total protein extracts were independently incubated with recombinant proteins of Ptr4CL3, Ptr4CL5, PtrHCT1, and PtrHCT6 with a C-terminal $6 \times$ His tag. The protein mixtures were affinity purified and analyzed by western blotting using anti-Ptr4CL3 (A) and anti-Ptr4CL5 (B) antibodies to identify interacting proteins. Recombinant proteins of Ptr4CL3, Ptr4CL5, PtrHCT1, PtrHCT6, and Gus were detected using His antibody (C). WB, western blot. Refer to Supplementary Figure $\mathbf{6}$ for the uncropped western blots.

using His-tag antibody (Figure 4C). The results of the pulldown assays provided further evidence that PtrHCT interacts with Ptr4CL to form protein complexes in vivo.

\section{Catalytic Activation of CoA-Ligation Activity in Ptr4CLs When Supplemented With PtrHCTs in vitro}

To investigate the role of PtrHCTs and their effects on CoAligation in a Ptr4CL-PtrHCT complex, we used recombinant proteins to study changes in the Ptr4CL activity when PtrHCT is supplemented in the reaction mixture. CoA ligation activity with 4-coumaric acid (Reaction A) and caffeic acid (Reaction B) as substrates were measured for mixed enzyme assays of Ptr4CL (10 $\mathrm{nM}$ ) and PtrHCT (40 nM). As a control, Ptr4CL reactions were supplemented with $40 \mathrm{nM}$ of bovine serum albumin (BSA). 
Ptr4CL3 activity was measured when supplemented with PtrHCT1 or PtrHCT6. Compared to Ptr4CL3 alone, the Ptr4CL3 activity toward 4-coumaric acid (Reaction A) increased by $43.4 \%$ when PtrHCT1 was added and by $40.9 \%$ when PtrHCT6 was added (Figure 5A). For caffeic acid (Reaction B), Ptr4CL3 activity increased by $39.7 \%$ and $36.6 \%$ when PtrHCT1 and PtrHCT6 were supplemented, respectively (Figure 5B). For Reaction $\mathbf{A}$ and Reaction $\mathbf{B}$, the Ptr4CL3 activities did not significantly change when BSA was added (Figures 5A,B). Similar to Ptr4CL3, the activity of Ptr4CL5 also increased when PtrHCT1 or PtrHCT6 was present. For Reaction A, the activity of Ptr4CL5 increased by $164.7 \%$ when PtrHCT1 was present and by $140.5 \%$ when PtrHCT6 was present (Figure 5C). For Reaction B, Ptr4CL5 activity increased by $124.2 \%$ when PtrHCT1 was present and by $113.8 \%$ when PtrHCT6 was present (Figure 5D). The addition of BSA to the Ptr4CL5 activity assays also did not alter the CoA ligation rate (Figures 5A-D).

The catalytic activations of Ptr4CLs by PtrHCTs in the in vitro enzyme assays are consistent with the findings that Ptr4CL activity is reduced when the expression of PtrHCTs was suppressed in the PtrHCT RNAi transgenic lines in vivo. These results indicate a regulatory role of the PtrHCT for Ptr4CL activities in the Ptr4CL-PtrHCT protein complex. However, the enhancement of Ptr4CL activities by PtrHCTs might be underestimated due to the extent of Ptr4CL-PtrHCT complex formation in the in vitro enzyme assays.

\section{Non-additive Co-enzyme A-Ligation Activity Due to PtrHCT Indicates a Specific Interaction Between Ptr4CL and PtrHCT}

Changes in enzyme activities mediated by the interactions between Ptr4CL and PtrHCT can be used to infer the stoichiometry of the protein complex. We investigated how Ptr4CL activities would affected the conversion of 4-coumaric acid (Reaction A) or caffeic acid (Reaction B) when the molar concentration of PtrHCT was varied (from 0 to $40 \mathrm{nM}$ ), while the molar concentration of Ptr4CL was fixed at $10 \mathrm{nM}$.

The CoA ligation activity when only Ptr4CL3 or Ptr4CL5 is present was taken as the baseline rate (dashed lines in Figure 6). The activities of Ptr4CL3 and Ptr4CL5 toward 4-coumaric acid (Reaction A) are shown in Figures 6A-D. The activities of both enzymes increased when the molar concentration of PtrHCTs became higher. The increasing activity plateaued when the Ptr4CL-PtrHCT ratio is 1:2, except for Ptr4CL5-PtrHCT1, yielding the highest activity with a ratio of 1:4 (Figure 6C). The activities of Ptr4CL3 and Ptr4CL5 toward caffeic acid (Reaction B) were also examined and shown in Figures 6E-H. Similar to the
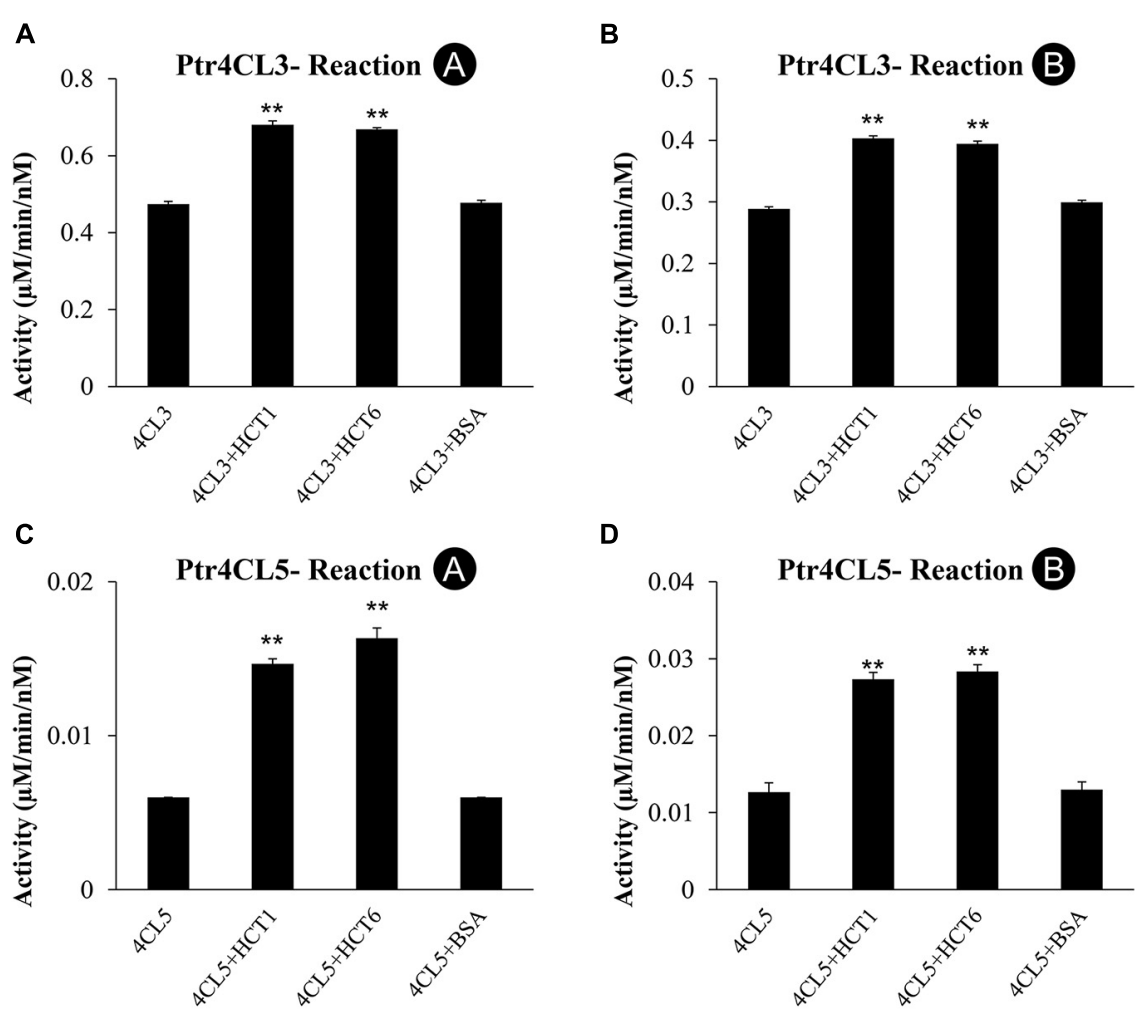

FIGURE 5 | CoA ligation activity with 4-coumaric acid (Reaction A) and caffeic acid (Reaction B) of recombinant Ptr4CL3 and Ptr4CL5 are affected by the presence of PtrHCT1 or PtrHCT6. (A) Reaction A of Ptr4CL3 with PtrHCTs. (B) Reaction B of Ptr4CL3 with PtrHCTs. (C) Reaction A of Ptr4CL5 with PtrHCTs. (D) Reaction B of Ptr4CL5 with PtrHCTs. Ptr4CLs were fixed at $10 \mathrm{nM}$, and PtrHCT concentration was fixed at $40 \mathrm{nM}$. Substrate concentration was fixed at $50 \mu \mathrm{M}$. Error bars represent one SE of three technical replicates. Statistical testing was performed using the Student's $t$-test $\left({ }^{\star \star} p<0.01\right)$. 
A

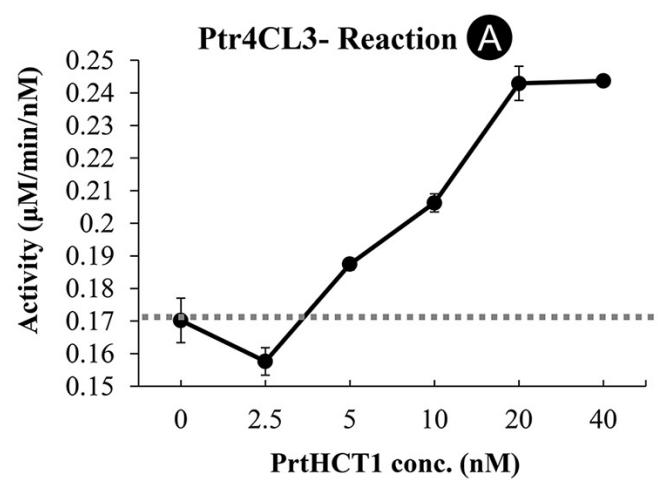

C

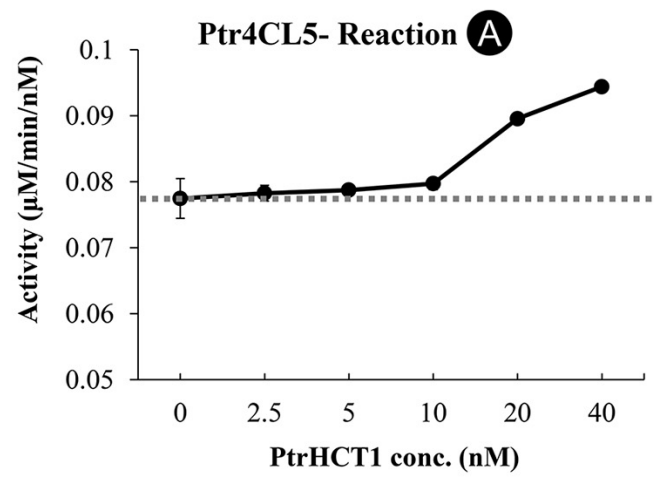

E

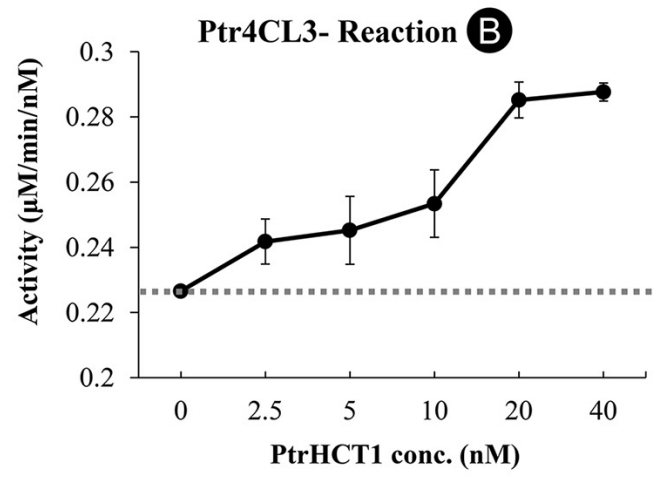

G

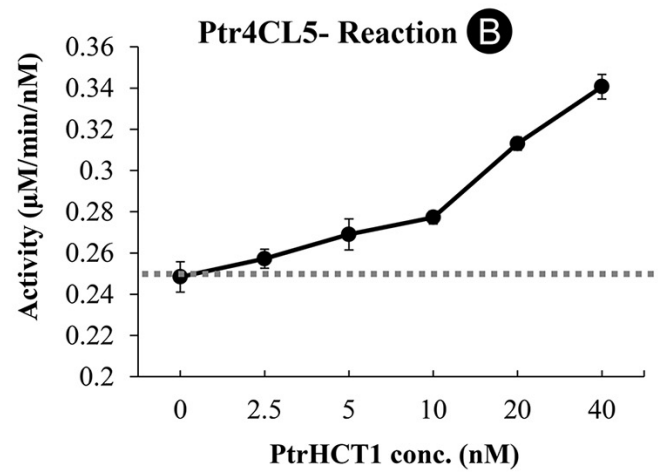

B

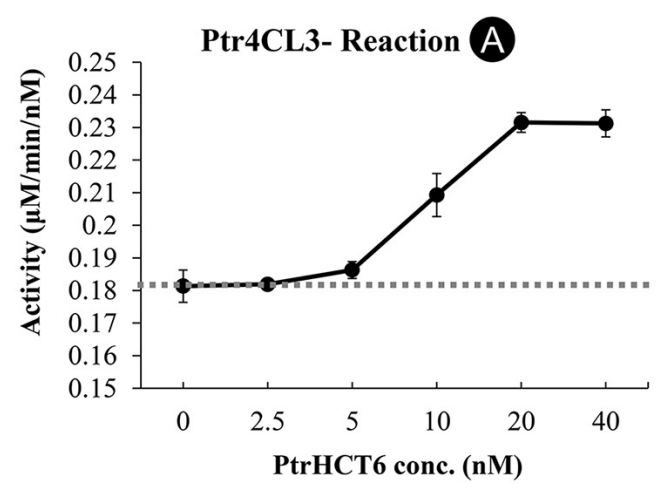

D

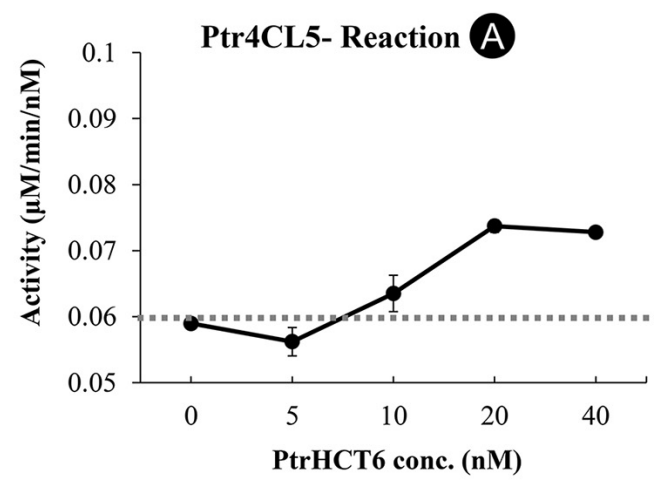

$\mathbf{F}$

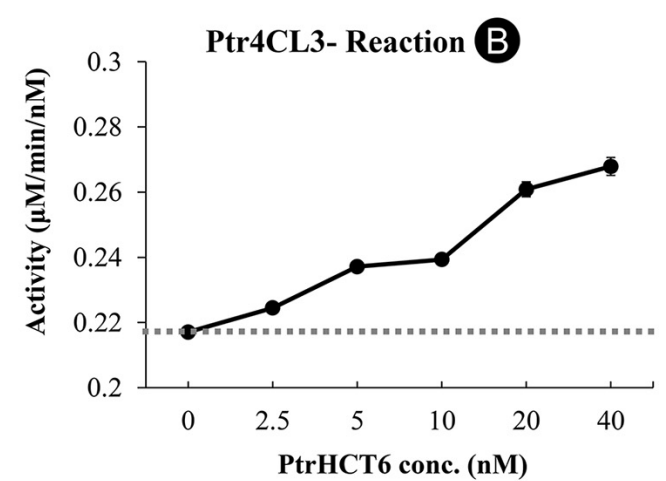

H

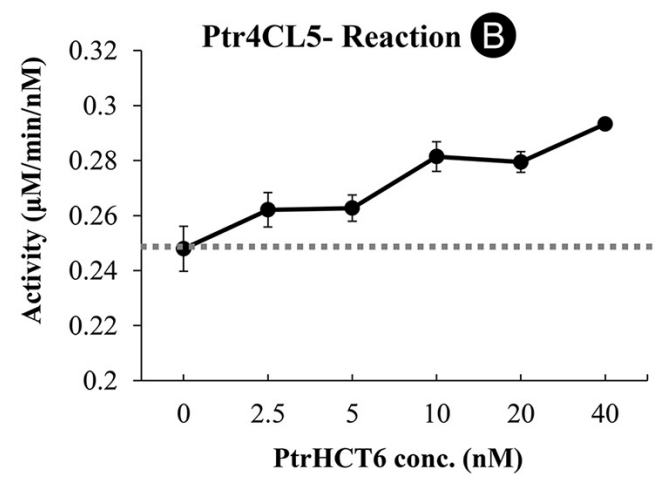

FIGURE 6 | Impact of Ptr4CL-PtrHCT complex formation on the CoA ligation of 4-coumaric acid (Reaction A) or caffeic acid (Reaction B). (A) Reaction A of Ptr4CL3 with increasing PtrHCT1 concentration. (B) Reaction A of Ptr4CL3 with increasing PtrHCT1 concentration. (C) Reaction A of Ptr4CL5 with increasing PtrHCT1 concentration. (D) Reaction A of Ptr4CL5 with increasing PtrHCT6 concentration. (E) Reaction B of Ptr4CL3 with increasing PtrHCT1 concentration. (F) Reaction B of Ptr4CL3 with increasing PtrHCT1 concentration. (G) Reaction B of Ptr4CL5 with increasing PtrHCT1 concentration. (H) Reaction B of Ptr4CL5 with increasing PtrHCT6 concentration. The concentration of Ptr4CL3 or Ptr4CL5 was fixed as $10 \mathrm{nM}$. Dashed lines indicate the baseline of activity of each Ptr4CL. Error bars represent SE of three replicates. 
A

Ptr4CL3- Reaction A

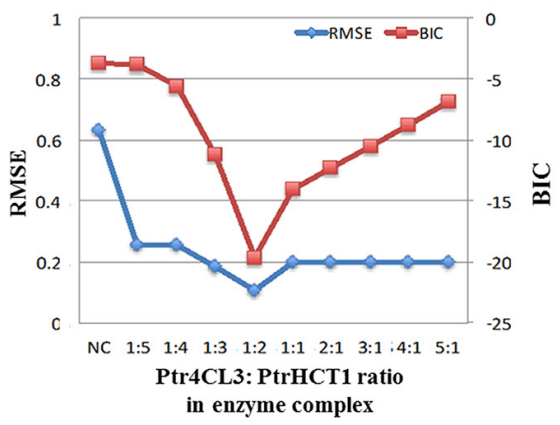

C

Ptr4CL5- Reaction A

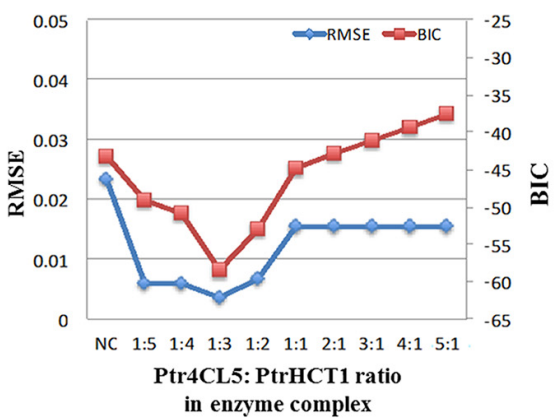

$\mathbf{E}$

Ptr4CL3- Reaction B

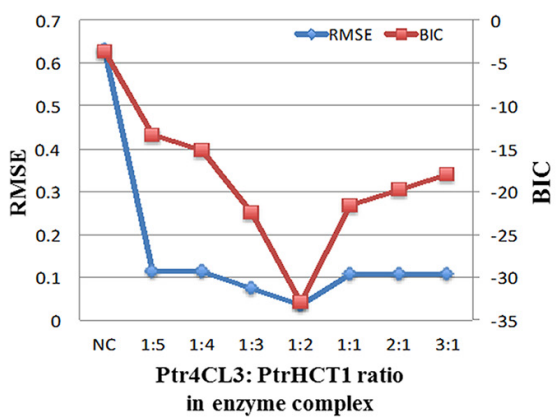

G

\section{Ptr4CL5- Reaction B}

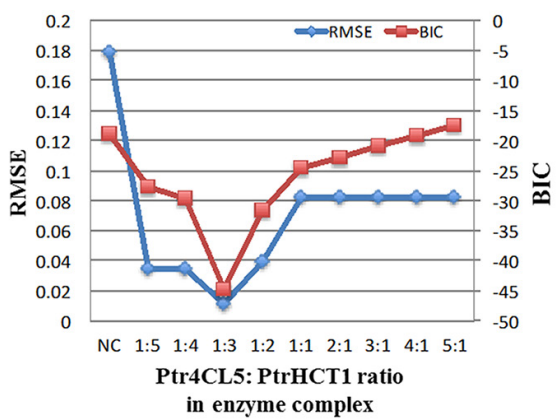

B

Ptr4CL3- Reaction A

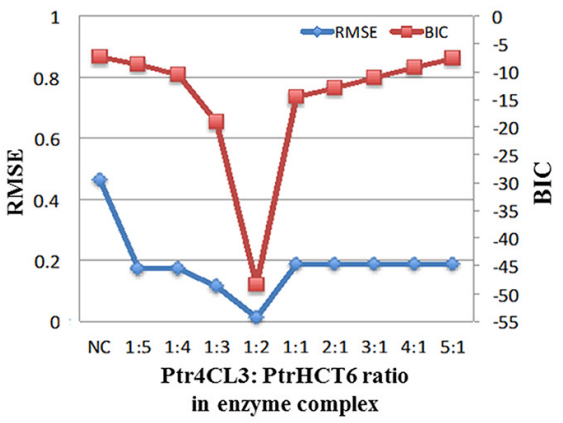

D

Ptr4CL5- Reaction A

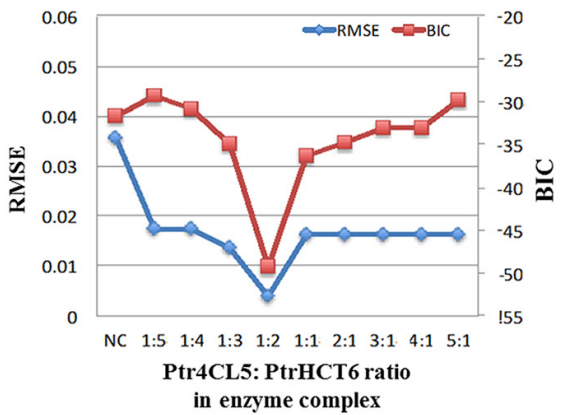

$\mathbf{F}$

Ptr4CL3- Reaction B

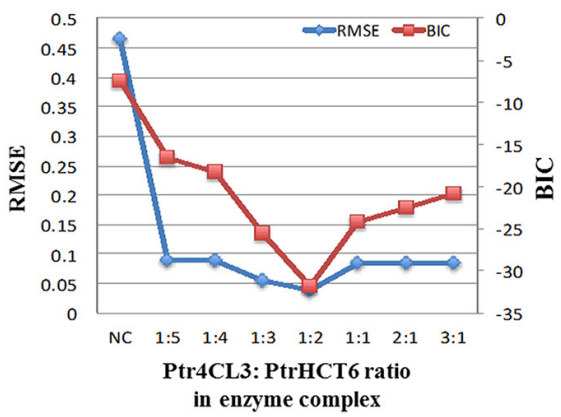

H

\section{Ptr4CL5- Reaction B}

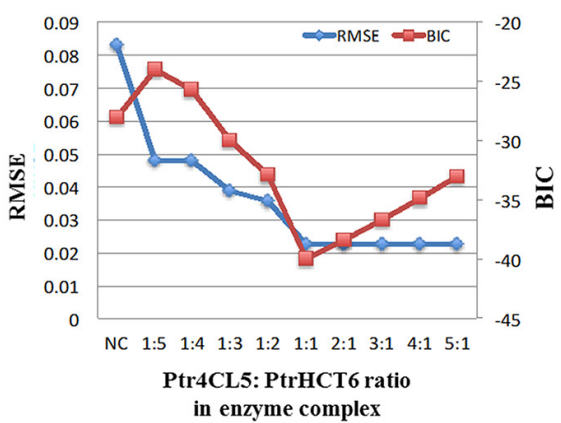

FIGURE 7 | Numerical analysis of CoA ligation activity for 4-coumaric acid (Reaction A) or caffeic acid (Reaction B) in model optimization process. (A) Ptr4CL3 to PtrHCT1 ratio for Reaction A. (B) Ptr4CL3 to PtrHCT6 ratio for Reaction A. (C) Ptr4CL5 to PtrHCT1 ratio for Reaction A. (D) Ptr4CL5 to PtrHCT6 ratio for Reaction A. (E) Ptr4CL3 to PtrHCT1 ratio for Reaction B. (F) Ptr4CL3 to PtrHCT6 ratio for Reaction B. (G) Ptr4CL5 to PtrHCT1 ratio for Reaction B. (H) Ptr4CL5 to PtrHCT6 ratio for Reaction B. RMSE, root mean squared error; BIC, Bayesian information criterion. 
case of Reaction A, both the activities of Ptr4CL3 and Ptr4CL5 for Reaction $\mathrm{B}$ were increased when the molar concentration of PtrHCTs became higher. An elevated CoA ligation activity was observed when the ratio of Ptr4CL and PtrHCT was around 1:2, except for Ptr4CL5 with PtrHCT6 that showed the elevated CoA ligation activity at 1:1 ratio (Figure $6 \mathbf{H}$ ). However, the plateau effect for caffeic acid is less evident than in the 4-coumaric acid reaction for both Ptr4CL3 and Ptr4CL5. Taken together, non-linear deviations from the Ptr4CL activity baseline when reactions were supplemented with PtrHCT were consistently observed (Figure 6). The non-additive increase in the CoAligation activities when Ptr4CL interacts with PtrHCT indicated a specific formation of the complex. These quantitative data were then used for mechanistic modeling and numerical analysis to investigate the stoichiometry of the Ptr4CL-PtrHCT complex.

\section{Mechanistic Modeling and Stoichiometry of Ptr4CL-PtrHCT Protein Complexes}

To explore the stoichiometry of the Ptr4CL-PtrHCT complex, a quantitative model was developed to describe how the Ptr4CLPtrHCT complex affects the overall reaction rate of Ptr4CL. The model was developed by a rule-based algorithm and an evolutionary computational optimization approach based on experimental estimates of enzyme activity (Supplementary Figure 4). Rule-based modeling can represent complex biochemical networks by building an interaction topology model between enzymes and substrates. The evolutionary computation method suggests the most suitable model through automatically mixing and matching potential interaction forms. This model contains both mechanistic modeling and numerical analysis, which has been successfully applied to reveal the functional redundancy of PtrCAld5Hs and the heterotetrameric interactions of the Ptr4CL complex (Wang et al., 2012; Chen et al., 2014; Song, 2014).

The model is based on experimental data and the mixed enzyme activity of Ptr4CLs in the Ptr4CL-PtrHCT complex (Figures 5, 6). The CoA ligation activities were given a fixed concentration of Ptr4CLs with different ratios of PtrHCTs toward 4-coumaric acid or caffeic acid were evaluated by numerical analysis according to the parameters for enzyme activity and enzyme ratios (Figure 6). The numerical analyses were used to measure the overall accuracy for stoichiometry predictions of the Ptr4CL-PtrHCT complex, evaluating the goodness-of-fit of models to the experimental data (Supplementary Figure 5). The model incorporated the enzyme activity under different ratios of Ptr4CL and PtrHCT and can indicate the stoichiometry of the complex based on the root mean square error (RMSE) and the Bayesian information criterium (BIC) (Schwarz, 1978). The parameters for optimizing the mechanistic modeling and the prediction of the stoichiometry of the Ptr4CL-PtrHCT complex were listed in Supplementary Table 2 toward 4-coumaric acid (Reaction A) and Supplementary Table 3 toward caffeic acid (Reaction B).

In most cases, both RMSE and BIC had the lowest values when the Ptr4CL to PtrHCT ratio is 1 to 2 (Figures 7A,B,D,E,F), except Ptr4CL5 showed a 1 to 3 ratio preference with

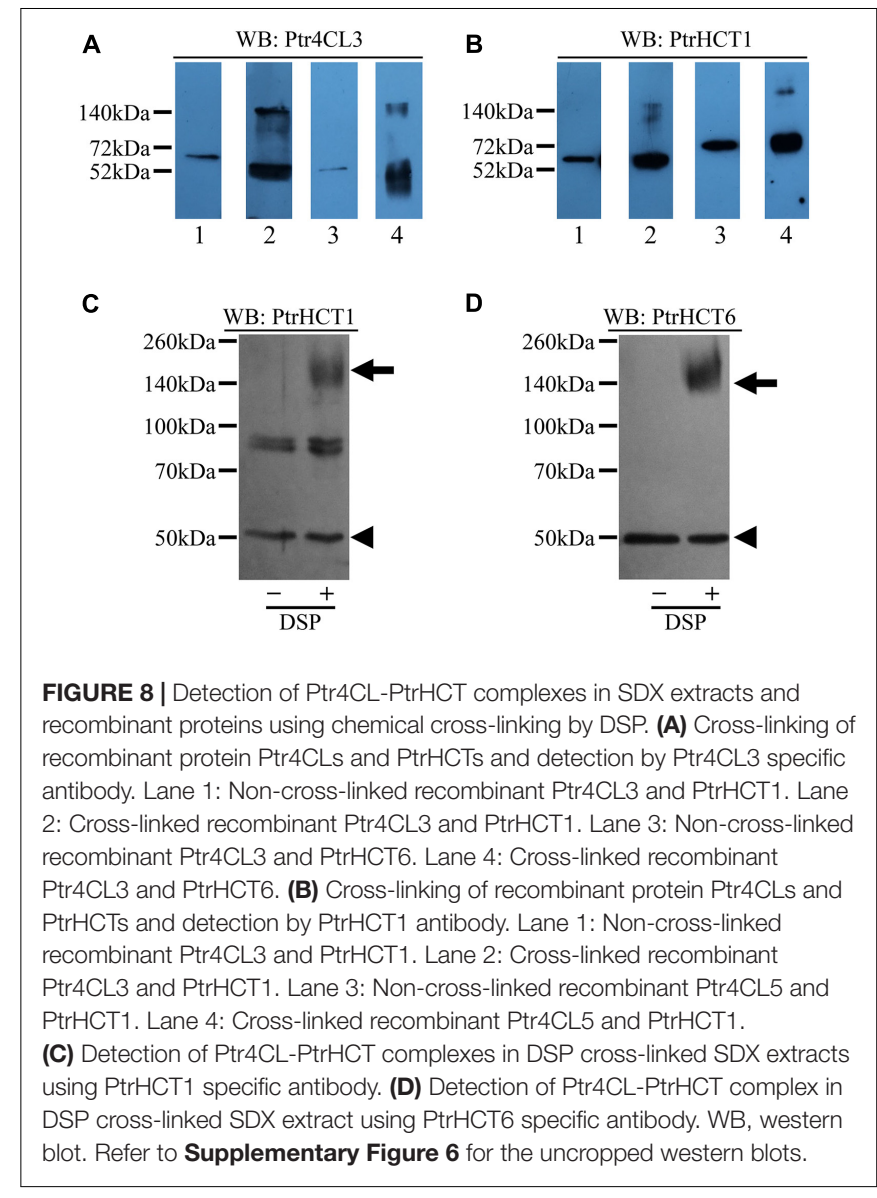

PtrHCT1 for Reaction A and Reaction B (Figures 7C,G) and a 1 to 1 ratio preference with PtrHCT6 for Reaction B (Figure $7 \mathbf{H}$ ). Although the numerical analysis indicates that the most likely protein complex is a heterotrimer of Ptr4CLPtrHCT with one subunit of Ptr4CL and two subunits of PtrHCTs, other possible combinations predicted for the Ptr4CL5PtrHCTs enzyme complex indicate there may be different specific interactions between Ptr4CLs and PtrHCTs when different isoforms become the predominant partners during monolingnol biosynthesis.

\section{The Ptr4CL-PtrHCT Complex Formation in vivo in SDX}

To obtain physical evidence for the stoichiometry of the Ptr4CLPtrHCT complex, we mixed recombinant proteins of Ptr4CL and PtrHCT with a chemical cross-linker, dithiobis [succinimidyl propionate] (DSP) (Lomant and Fairbanks, 1976). The crosslinking of PtrHCT and Ptr4CL recombinant proteins was carried out with equal concentration $(200 \mathrm{nM})$ of the interacting proteins. The cross-linked proteins were then resolved by SDSPAGE, and detected using protein-specific antibodies. Using an anti-Ptr4CL3 antibody, Ptr4CL3 without cross-linking showed a protein band of $\sim 60 \mathrm{kDa}$, consistent with the molecular weight of a monomeric unit of Ptr4CL3 (Figure 8A, Lines 1 and 3). Crosslinking of Ptr4CL3 with PtrHCT1 or PtrHCT6 (Figure 8A, Lines 
2 and 4$)$ revealed a much larger protein band $(\sim 160 \mathrm{kDa})$ in addition to the monomer, suggesting the presence of a protein complex. Similarly, using PtrHCT1 specific antibody for western blot detection, a protein band $(\sim 160 \mathrm{kDa})$ was detected after cross-linking PtrHCT1 with Ptr4CL3 or Ptr4CL5 (Figure 8B, Lines 2 and 4$)$. The protein band $(\sim 160 \mathrm{kDa})$ approximates the molecular weight of the Ptr4CL-PtrHCT complex at a 1:2 ratio predicted by the numerical analysis.

Furthermore, to obtain physiological evidence for the Ptr4CLPtrHCT complex in vivo, we investigated the existence of the Ptr4CL-PtrHCT complex in crude SDX extracts of $P$. trichocarpa. The SDX extracts were cross-linked using DSP, resolved by SDSPAGE, and detected using anti-PtrHCT antibodies. Compared to SDX extracts without chemical cross-linking, a protein band of $\sim 160 \mathrm{kDa}$ was detected using PtrHCT1 specific antibody (Figure 8C), as well as PtrHCT6 specific antibody (Figure 8D). The estimated size of the bands is consistent with the expected size of the heterotrimeric Ptr4CL-PtrHCT complex for one unit of Ptr4CL and two units of PtrHCT. These results confirmed the presence of a Ptr4CL-PtrHCT complex when both proteins are present in the recombinant protein mixtures in vitro and in the SDX in vivo. The stoichiometry of the Ptr4CL-PtrHCT complex is likely to be a 1 to 2 ratio.

\section{DISCUSSION}

In this study, we provide several lines of evidence for a Ptr4CL-PtrHCT complex in P. trichocarpa, including (1) PtrHCT RNAi downregulated transgenic trees (Figure 2), (2) pairwise combinations of BiFC (Figure 3), (3) protein pull-down from SDX extracts (Figure 4) and (4) enzyme activity using recombinant proteins (Figures 5, 6). By conducting the rulebased modeling and evolutionary computation, the most likely composition of the Ptr4CL and PtrHCT complex is predicted to have one subunit of Ptr4CL and two subunits of PtrHCTs (Figure 7). Finally, from chemical cross-linking of recombinant proteins and SDX crude extracts a protein band of $\sim 160 \mathrm{kDa}$ was detected, which confirmed the predicted stoichiometry of a Ptr4CL-PtrHCT complex (Figure 8). In addition to the identification of protein-protein interactions between Ptr4CL and PtrHCT, the presence of PtrHCTs can facilitate the CoA ligation activity of Ptr4CLs for both 4-coumaric acid and caffeic acid (Figures 5, 6). This complex could play an important role in regulating the metabolic flux for CoA ligation in monolignol biosynthesis.

\section{Evidence of a 4CL-HCT Complex and Its Physiological Function}

In Arabidopsis, the three isoforms of 4CL possess different substrate preferences and expression patterns. At4CL1 was proposed to be the best candidate for lignin formation (Ehlting et al., 1999). At the early branch point of monolignol biosynthesis in Arabidopsis, AtHCT was found partially associated with the ER and connected to the membrane-bound CYP73A5 (AtC4H) and CYP98A3 (AtC3' $\mathrm{H}$ ) (Bassard et al., 2012). Moreover, AtC3' $\mathrm{H}$ was proposed to be the most effective in driving protein association with At4CL1 and AtHCT to the ER, while wound repair from mechanical injury could enhance the re-localization of these enzymes (Bassard et al., 2012). Neither TAP tagging nor blue native gel mobility could detect the association of At4CL1 and AtHCT in their native forms (Zhang and Liu, 2015); therefore, the physiological role of the 4CL-HCT association in Arabidopsis has not yet been established.

It has been suggested that a phenylpropanoid metabolon could alter the enzyme activity of different isoforms of monolignol biosynthetic enzymes (Jorgensen et al., 2005). In stem-differentiating xylem (SDX) of P. trichocarpa, two Ptr4CL isoforms (Ptr4CL3 and Ptr4CL5) and two PtrHCT isoforms (PtrHCT1 and PtrHCT6) were identified as xylem-specific and xylem-abundant enzymes that participate in monolignol biosynthesis (Shi et al., 2010). First, the reciprocal BiFC assays supported the protein-protein interaction between the Ptr4CLs and PtrHCTs (Figure 3). Then, we detected the direct proteinprotein interactions between the isoforms of Ptr4CL and PtrHCT using pull-down assays (Figure 4). Consistently, the interaction between the isoforms of Ptr4CL and PtrHCT was shown to facilitate the enzyme activity of $4 \mathrm{CL}$ to promote CoA-ligation (Figures 5, 6).

Numerical analyses of the CoA ligation activity predicted a 12 Ptr4CL to PtrHCT protein ratio in the complexes (Figure 7). Besides the predominately predicted 1:2 ratio of Pt4CL-PtrHCT complex, there are other possible combinations predicted for the Ptr4CL5-PtrHCTs enzyme complex, such as a 1-3 ratio for Ptr4CL5-PtrHCT1 complex (Figures 7C,G) and a 1-1 ratio for Ptr4CL5-PtrHCT6 complex (Figure $7 \mathbf{H}$ ), indicating that some specific interactions between Ptr4CL5 and PtrHCTs were able to be captured by our numerical analyses when different isoforms dominate in a well-controlled environment. However, a 1:2 ratio of Pt4CL-PtrHCT complex is experimentally confirmed by chemical cross-linking using SDX plant protein extracts (Figure 8).

An alternative role for the interaction between Ptr4CL and PtrHCT may be to protect 4-coumaroyl-CoA without it being released into the cytosolic pool, considering that the CoA thioesters, such as 4-coumaroyl-CoA, are relatively unstable metabolites and can be easily hydrolyzed in protein extracts (Loscher and Heide, 1994; Liang et al., 2010). The formation of a Ptr4CL-PtrHCT complex may facilitate the synthesis of CoA thioesters while further directing metabolic flux toward downstream monolignol biosynthesis without competing with other pathways. Moreover, in sorghum (Sorghum bicolor), a structural analysis of the SbHCT shows a significant affinity of SbHCT for 4-coumaroyl-CoA $(1.6 \mu \mathrm{M})$ and a negligible affinity for shikimic acid, which suggests that 4-coumaroyl-CoA preconditions the binding of shikimic acid to SbHCT (Walker et al., 2013). Therefore, the Ptr4CL-PtrHCT complex could ensure the efficient binding of CoA thioesters to PtrHCT.

\section{Protein-Protein Interactions in Phenylpropanoid Metabolism}

There are well-described mechanisms in the secondary metabolism of plants to effectively produce specific 
metabolites while avoiding metabolic interference, such as compartmentation, metabolic channeling, and metabolon formation (i.e., multienzyme complexes) (Ovádi, 1991; Jorgensen et al., 2005; Zhang and Fernie, 2020). These mechanisms can possess physiologically significant regulatory roles, such as relief of kinetic constraints, regulation of catalytic efficiency, sequestration of labile/toxic intermediates, and coordination of metabolic crosstalk to control the flux of metabolic sequences (Winkel-Shirley, 2001; Nakayama et al., 2019). These regulatory controls can facilitate the effective utilization of intermediates and modulate the carbon/energy flux among the branched pathways that often function concurrently in the same cell (Luckner, 1984; Weid et al., 2004; Winkel, 2004; Jorgensen et al., 2005; Ralston and Yu, 2006; Laursen et al., 2015; Bassard and Halkier, 2018). Metabolons are typically described as protein-protein interactions between 'soluble' enzymes that might be anchored to biological membranes or cytoskeletal elements, either by structural proteins or membrane-bound "anchor" proteins such as P450s. Early studies on proteinprotein interactions suggested metabolite compartmentation or metabolic channeling in flavonoid biosynthesis (Fritsch and Grisebach, 1975; Jacques et al., 1977; Hrazdina et al., 1978; Stafford, 1981), isoflavonoids (Dixon et al., 1998; He and Dixon, 2000; Liu and Dixon, 2001; Waki et al., 2016), and monolignols (Czichi and Kindl, 1975, 1977; Hrazdina and Wagner, 1985b). For flavonoid biosynthesis, several members within specific metabolons were identified in different plant species, indicating the existence of the functional flavonoid metabolons (Dastmalchi et al., 2016; Fujino et al., 2018; Mameda et al., 2018; Nakayama et al., 2019).

In 1985, an ER-associated multienzyme complex for monolignol biosynthesis was proposed (Stafford, 1974b). Later, metabolic complexes for $\mathrm{G}$ and $\mathrm{S}$ monolignol biosynthesis were suggested, while COMT and CCoAOMT/CCR were thought to have independent metabolic routes (Dixon et al., 2001). Physical evidence for separate monolignol complexes is lacking, and the identification of lignin metabolons remains ongoing. Several protein complexes had been identified in monolignol biosynthesis, including a PAL-C4H complex (Rasmussen and Dixon, 1999; Achnine et al., 2004), a MSBP/P450 complex (Gou et al., 2018), a C3'H/C4H complex (Chen et al., 2011), a 4CL complex (Chen et al., 2014), and a CAD/CCR complex (Yan et al., 2019). In contrast to flavonoid biosyntheses, the regulatory effects of protein complexes in monolignol biosynthesis may support mechanisms other than metabolic channeling (Pettersson, 1991), such as feedback inhibition of PAL by cinnamate (Blount et al., 2000), the biochemical coupling of PAL-C4H (Ro and Douglas, 2004), and the absence of substrate channeling through a MSBP/P450 complex (Zhang and Fernie, 2020). In P. trichocarpa, the evidence of metabolic channeling remains debatable because exogenous metabolites can be used by the $\mathrm{C}^{\prime} \mathrm{H} / \mathrm{C} 4 \mathrm{H}$ complex, and all 24 endogenous monolignol precursors can be detected in the crude SCW protein extract (Chen et al., 2011).

\section{Identification of a Lignin Metabolon}

In flavonoid biosynthesis, the interactions among proposed metabolon enzymes vary between plant species, implying species-dependent metabolon formations for the diverse phenylpropanoids in planta, especially flavonoids (Nakayama et al., 2019). The flavonoid metabolon identified in snapdragon involved all FNSII, CHS, CHI, and DFR enzymes, while, in torenia, CHS was not found to interact with the proposed enzymes within the metabolon (Fujino et al., 2018). In hops, an active membrane-anchored metabolon for xanthohumol (prenylated flavonoid) biosynthesis has recently been identified. CHIL2, a non-catalytic CHI-like protein (CHIL), interacts with CHS_H1 (a hop specific CHS) and a membrane-bound prenyltransferase (PT1L) (Ban et al., 2018). Using Förster resonance energy transfer detected by fluorescence lifetime imaging microscopy (FLIM-FRET) in Arabidopsis protoplasts, CHS was identified to interact with flavonol synthase 1 (FLS1) or DFR to organize the flavonoid metabolon in a competitive manner to further modulate the metabolic flux into branching pathways (Crosby et al., 2011). Similarly, the identified enzyme complex of Ptr4CL-PtrHCT can be formed in a collaborative manner with the previously identified Ptr4CL3-Ptr4CL5 complex (Chen et al., 2014) or in a competitive mechanism in the lignin metabolon to modulate the CoA ligation flux for monolignol biosynthesis. The evidence of Ptr4CL-PtrHCT complex formation could be useful to investigate the possible role of these complexes or could be further integrated into a metabolic flux analysis for the identification of a lignin metabolon.

Moreover, crosstalk between metabolic enzymes among different metabolons should also be considered. In the CHI Arabidopsis flavonoid mutant (tt5), deficient for the major extractable leaf flavonols, the simultaneous downregulation of the sinapate ester biosynthesis was also observed even with the full capacity of the monolignol pathway (Li et al., 1993). For the production of isoflavonoids in soybean roots, GmCHR5, one of the $11 \mathrm{GmCHR}$ paralogs, is the key enzyme for the physiological accumulation of 5-deoxyisoflavonoids by spatialspecific incorporation into the flavonoid metabolon (Mameda et al., 2018). Two other paralogs of GmCHR, GmCHR1, and GmCHR6, are proposed to involve the production of isoflavonoids for defense against microbial pathogens (Nakayama et al., 2019). Of the 17 Ptr4CL genes identified in the genome of P. trichocarpa, Ptr4CL1 and Ptr4CL17 also showed some specificity for differentiating xylem (Shi et al., 2010), despite their low transcript levels. These low abundance Ptr4CL paralogs could also participate in the lignin metabolon, which should be explored along with the Ptr4CL3 and Ptr4CL5. Moreover, the crosstalk between flavonoid and lignin metabolons is also possible by sharing a similar intermediate metabolite, 4coumaroyl-CoA. Ptr4CL4 in P. trichocarpa (Shi et al., 2010) and 4CL2 in P. tremuloides (Hu et al., 1998) have both been associated with the biosynthesis of flavonoids and other soluble phenolics, which could be candidates for future investigation of protein-protein interactions.

The isolation of intact metabolons can be challenging if the protein-protein interactions in metabolons are weak or transient (Jorgensen et al., 2005; Nakayama et al., 2019). PAL-C4H is mediated by weak interactions that easily disassociate depending on the integrity of membranes during the purification (Hrazdina, 1992), treatment with ethylene (Czichi and Kindl, 1975), or under different physiological states (Luckner, 1984). In 
Arabidopsis, a loose association of P450s with HCT and 4-CL1 was observed (Bassard et al., 2012). Using an $\mathrm{Y} 2 \mathrm{H}$ assay, At4CL1 had a physical interaction with AtC4H and $\mathrm{AtC}^{\prime} \mathrm{H}$, while AtCCR1 interacted with AtC4H (Gou et al., 2018). In $P$. trichocarpa, four monolignol protein complexes were identified, including PtrC3 ${ }^{\prime} \mathrm{H}-\mathrm{PtrC} 4 \mathrm{H}$, Ptr4CL3-Ptr4CL5, Ptr4CL-PtrHCT, and PtrCAD-PtrCCR. However, the complex formations for other monolignol biosynthetic enzymes, such as COMT, CCoAOMT, have not yet been linked to a lignin metabolon, and the relationships between the enzyme complex during the early steps of monolignol biosynthesis and the later steps such as F5H for downstream G- and S- monolignol biosynthesis remain unknown.

In the past, traditional techniques were conducted to illustrate the possible membrane-bound ER-resident protein complexes of phenylpropanoid metabolism, such as feeding labeled intermediates, cellular fractionation, co-immunolocalization, and gene co-expression analysis (Winkel-Shirley, 1999). Several successful techniques for studying protein-protein interactions could also be useful for future endeavors to investigate the lignin metabolon (Bassard and Halkier, 2018), such as two-hybrid assay (Ehlert et al., 2006), luciferase complementation imaging assay (LuCIA) (Ban et al., 2018), cross-linking/mass spectrometry (XL-MS) (Tang and Bruce, 2010), enzyme reconstitution (MorkJansson and Eichacker, 2019), microencapsulation (Anderson, 1974), and cryo-electron microscopy (cryo-EM) (Du et al., 2018). To reveal whether the existence of a cell-type-specific or a G/Sspecific lignin metabolon is an intriguing topic for understanding the specific lignin deposition among different plant species. Metabolons can also vary greatly in physical stability, and the difference between an enzyme that presents in a defined stoichiometric ratio and a metabolon is neither precise nor absolute (Jorgensen et al., 2005). There are still many unanswered questions that need to be explored, including the possibility of an interactome among all the monolignol biosynthetic enzymes, regulatory effects on metabolic flux between phenylpropanoid metabolisms, dynamic compositions of the lignin metabolon for differential monolignol deposition, and utilization of the isoforms of monolignol biosynthetic enzymes in response to biotic and abiotic stresses.

\section{CONCLUSION}

In the phenylpropanoid pathway, both flavonoid and monolignol biosyntheses are based on enzymes catalyzing sequential reactions. It is reasonable to investigate the spatial separation, competition, crosstalk, and affinities between the monolignol biosynthetic enzymes together with the intracellular distribution/transportation of the metabolites. These factors should play essential roles in modulating the functions of the monolignol enzyme complexes, leading to distinct regulatory and physiological roles in a lignin metabolon (Biała and Jasiński, 2018; Nakayama et al., 2019). Besides the previously identified monolignol complexes in planta, in this study, we provide direct evidence of a Ptr4CL-PtrHCT complex, which we propose to exist in $P$. trichocarpa for further modulating the CoA-ligation toward monolignol biosynthesis. The current knowledge is still far from sufficient to reconstruct a lignin metabolon. Efforts to unravel the supramolecular structure and function of this intriguing metabolon can shed some light on the enduring mystery of monolignol biosynthesis in different tissue types, differential lignin compositions, and the metabolic switches for specific metabolites in planta. A basic understanding of a monolignol metabolon could provide new strategies to manipulate the phenylpropanoid pathway to generate high-quality biomass for biofuel and other bioproducts.

\section{MATERIALS AND METHODS}

\section{Plant Materials}

Clonal propagules of Populus trichocarpa (Nisqually-1) wildtype and the PtrHCT RNAi transgenic lines by Wang et al. (2018) were grown in a greenhouse in soil containing a 1:1 ratio of peat moss to potting-mix, using a $16 \mathrm{~h}$ light and $8 \mathrm{~h}$ dark photoperiod for 6 months before harvesting. The stem differentiating xylem (SDX) tissues were collected by scraping debarked stems using single-edge razor blades and instantly frozen in liquid nitrogen before storage at $-80^{\circ} \mathrm{C}$.

\section{Quantification of Transcripts and Proteins of Ptr4CLs and PtrHCTs in Stem-Differentiating Xylem of PtrHCT-RNAi Transgenic Lines and Wildtype}

The quantitative data (transcript and protein abundances) of Ptr4CLs (Ptr4CL3 and Ptr4CL5) and PtrHCTs (PtrHCT1 and PtrHCT6) from the PtrHCT RNAi transgenic lines and the corresponding WT controls were retrieved from SDX transcriptomes (Wang et al., 2018) and absolute protein quantification from SDX crude protein extracts using protein cleavage isotope dilution mass spectrometry (PC-IDMS) analysis (Shuford et al., 2012). For WT or each line of transgenic plant, a biological replicate represents a pool of three to five clonally propagated trees.

\section{Enzyme Activity Assays of Ptr4CL in Stem-Differentiating Xylem Extracts}

Stem-differentiating xylem crude protein extractions of WT and PtrHCT RNAi transgenic lines were conducted following our established protocols (Shi et al., 2010; Lin et al., 2015; Wang et al., 2018). Three grams of SDX was ground, extracted by ice-cold buffer (50 mM Tris- $\mathrm{HCl}, \mathrm{pH}$ 7.5, $20 \mathrm{mM}$ sodium ascorbate, 0.4 M sucrose, $100 \mathrm{mM} \mathrm{NaCl,} 5 \mathrm{mM}$ DTT, 10\% PVPP, $1 \mathrm{mM}$ PMSF, $1 \mathrm{mg} / \mathrm{mL}$ pepstatin A and $1 \mathrm{mg} / \mathrm{mL}$ leupeptin), homogenized for $2 \mathrm{~min}$ on ice, and cleared twice by centrifugation at $4,000 \times g$ for $15 \mathrm{~min}$ at $4^{\circ} \mathrm{C}$. The supernatant was filtered, quantified the protein concentration of SDX extracts by the Bradford method, and stored at $-80^{\circ} \mathrm{C}$ before enzyme assays.

Conditions for enzyme assays of Ptr4CL activities using SDX crude protein extracts also followed our established protocol (Liu et al., 2012). In brief, the activity assays of Ptr4CL reactions 
were examined using $20 \mu \mathrm{g}$ SDX protein extracts at $37^{\circ} \mathrm{C}$ for 30 min with $50 \mu \mathrm{M}$ substrate (final concentration) in the assay solution [50 mM Tris- $\mathrm{HCl}$ buffer ( $\mathrm{pH} 7.5$ ), $2.5 \mathrm{mM} \mathrm{MgCl}_{2}, 5 \mathrm{mM}$ ATP, $0.2 \mathrm{mM}$ CoA] to a final volume of $100 \mu \mathrm{L}$. The reaction was terminated by the addition of $5 \mu \mathrm{L}$ of $3 \mathrm{M}$ trichloroacetic acid (TCA). Each reaction was repeated three times. After the mixture was centrifuged at $20,000 \times g$ for $20 \mathrm{~min}, 90 \mu \mathrm{L}$ of supernatant reaction mixture was transferred to an HPLC sample vial and $75 \mu \mathrm{L}$ of supernatant was injected directly for HPLC analysis following (Liu et al., 2012).

\section{Purification of Recombinant Proteins and in vitro Enzyme Assays of Ptr4CLs}

The procedures for expressing and purifying individual recombinant proteins (Ptr4CL3, Ptr4CL3, PtrHCT1, or PtrHCT6) and in vitro enzyme assays of Ptr4CLs were performed as previously described (Wang et al., 2014; Lin et al., 2015). In brief, a $100 \mu \mathrm{L}$ reaction containing final concentration of $50 \mu \mathrm{M}$ substrate (4-coumaric acid or caffeic acid), $0.2 \mathrm{mM} \mathrm{CoA}$, $5 \mathrm{mM}$ ATP and $2.5 \mathrm{mM} \mathrm{MgCl}_{2}$ in $50 \mathrm{mM}$ Tris- $\mathrm{HCl}$ buffer $(\mathrm{pH}$ 8.0 for Ptr4CL3 and $\mathrm{pH} 7.0$ for Ptr4CL5) starts with a final enzyme concentration of $10 \mathrm{nM}$ of purified recombinant Ptr4CLs for a reaction time of $10 \mathrm{~min}$ at $40^{\circ} \mathrm{C}$. The concentrations of recombinant PtrHCT in the enzyme assays were indicated in the corresponding figure legends.

\section{Bimolecular Fluorescence Complementation Assay}

The coding sequences of PtrHCT6, PtrHCT1, Ptr4CL3, Ptr4CL5 without the stop codons were obtained by PCR using specific primer sets (Supplementary Table 1) and cloned using the $\mathrm{pENTR}^{\mathrm{TM}} / \mathrm{D}-\mathrm{TOPO}^{\mathrm{TM}}$ Cloning Kit (K240020, Invitrogen Carlsbad, CA, United States), and then recombined into the BiFC destination vector. All plasmids for bimolecular fluorescence complementation (BiFC) were prepared by $\mathrm{CsCl}$ gradient ultracentrifugation (Meselson et al., 1957; Radloff et al., 1967; Sambrook et al., 1982; Tartof, 1987; Sambrook and Russell, 2006). Each pair of the enzyme genes (Ptr4CL3$\mathrm{YFP}^{\mathrm{N}}+$ PtrHCT1-YFPC, Ptr4CL3-YFP + PtrHCT6-YFP Ptr4CL3-YFP ${ }^{\mathrm{N}}+$ Ptr4CL3-YFPC, Ptr4CL3-YFP ${ }^{\mathrm{N}}+$ Ptr4CL5$\mathrm{YFP}^{\mathrm{C}}, \quad$ Ptr4CL5-YFP ${ }^{\mathrm{N}}+$ PtrHCT1-YFP $^{\mathrm{C}}$, Ptr4CL5-YFP ${ }^{\mathrm{N}}$ + PtrHCT6-YFPC , Ptr4CL5-YFP ${ }^{\mathrm{N}}+$ Ptr4CL3-YFPC, Ptr4CL5$\mathrm{YFP}^{\mathrm{N}}+$ Ptr4CL5-YFP ${ }^{\mathrm{C}}$, PtrHCT1-YFP ${ }^{\mathrm{N}}+$ PtrHCT1-YFPC $^{\mathrm{C}}$, PtrHCT1-YFP + PtrHCT6-YFPC ${ }^{\mathrm{N}}$ PtrHCT1-YFP ${ }^{\mathrm{N}}+$ Ptr4CL3$\mathrm{YFP}^{\mathrm{C}}$, PtrHCT1-YFP ${ }^{\mathrm{N}}+$ Ptr4CL5-YFPC, PtrHCT6-YFP ${ }^{\mathrm{N}}$ + PtrHCT1-YFPC, PtrHCT6-YFP ${ }^{\mathrm{N}}+$ PtrHCT6-YFPC $^{\mathrm{C}}$, PtrHCT6-YFP ${ }^{\mathrm{N}}+$ Ptr4CL3-YFPC , PtrHCT6-YFP ${ }^{\mathrm{N}}+$ Ptr4CL5$\mathrm{YFP}^{\mathrm{C}}$ ) were co-transfected into the SDX protoplasts. As negative controls (Ptr4CL3-YFP ${ }^{\mathrm{N}}+$ Gus-YFPC $^{\mathrm{C}}$, Ptr4CL5$\mathrm{YFP}^{\mathrm{N}}+$ Gus-YFPC, PtrHCT1-YFP ${ }^{\mathrm{N}}+$ Gus-YFPC $^{\mathrm{C}}$, PtrHCT6-YFP ${ }^{\mathrm{N}}+$ Gus-YFPC) were co-transfected into the SDX protoplasts.

The transient expression of BiFC in SDX protoplasts followed our previously established protocol (Lin et al., 2014). After incubation for $12 \sim 16 \mathrm{~h}$, SDX protoplasts were collected and examined under a ZEISS LSM 700 fluorescence microscope. The excitation wavelength and the emission wavelength were 515 and $525 \mathrm{~nm}$, respectively.

\section{Pull-Down Assays}

Coding sequences of Ptr4CL3, Ptr4CL5, PtrHCT1, and PtrHCT6 without stop-codons were transferred from the pENTR gateway vector into pET101-DEST (C-terminal $6 \times$ His tag) expression vector (K10101, Invitrogen) to generate the pET101::Gene$6 \times$ His plasmids for recombinant protein expression in Escherichia coli. The procedures for the expression and purification of recombinant proteins were previously described (Wang et al., 2014; Lin et al., 2015). A pET101:Gus-6 × His control was included with each pull-down to detect non-specific binding proteins (Liu et al., 2012; Wang et al., 2012; Chen et al., 2013). Recombinant $6 \times$ His fused monolignol enzymes $(60 \mu \mathrm{g})$ were individually incubated as bait protein in $50 \mathrm{~mL}$ of crude SDX protein extracts $(0.4 \mathrm{mg} / \mathrm{mL})$ at $4^{\circ} \mathrm{C}$ for $2 \mathrm{~h}$. The protein mixtures were then affinity purified using immobilized nickel affinity chromatography to isolate interacting proteins bound to the recombinant monolignol bait protein. The affinity-purified proteins were analyzed by western blotting using polyclonal anti-Ptr4CL3, anti-Ptr4CL5, anti-PtrHCT1, and anti-PtrHCT6 antibodies as previously described (Chen et al., 2014).

\section{Mechanistic Modeling and Numerical Analysis}

Mechanistic modeling was carried out by a previously developed rule-based algorithm and evolutionary computation algorithm for monolignol enzymes (Chen et al., 2014; Song, 2014). The algorithmic evolutionary processes automatically search and optimize the best solution for complex non-linear problems (Supplementary Figure 4). The method identifies possible components and interactions in multi-enzymatic reactions and suggests model structures and mathematical equations representing the mechanism of multi-enzymatic reactions.

The model is based on the experimental results of Ptr4CL CoA ligation reactions in mixtures of Ptr4CL and PtrHCT and evaluated by the numerical analysis of evolutionary computation. Two different numerical methods were used, the root mean squared error (RMSE) and Bayesian information criterion (BIC). The lower values of RMSE and BIC indicate the better models. The differences between the predicted values and experimental values are measured by RMSE. The lower values of RMSE and $\mathrm{BIC}$ indicate the better models. Adding on the goodness-of-fit of the model, BIC also reflects the model complexity using a penalty term for the number of parameters (Schwarz, 1978).

\section{Chemical Cross-Linking of Stem-Differentiating Xylem Proteins and Recombinant Proteins}

Dithiobis (succinimidyl propionate) (DSP) (22585, Thermo Scientific, Waltham, MA, United States) was used as the crosslinker for the detection of protein-protein interactions. A $50 \mathrm{mM}$ solution of DSP cross-linker was prepared by dissolving $10 \mathrm{mg}$ of DSP in $495 \mu \mathrm{L}$ of DMSO. 
For cross-linking of SDX proteins, the SDX proteins were extracted using $25 \mathrm{mM}$ sodium phosphate buffer ( $\mathrm{pH}$ 7.4) instead of the $50 \mathrm{mM}$ Tris- $\mathrm{HCl}$ buffer. The detailed cross-linking process of SDX proteins was previously described (Chen et al., 2011). For cross-linking of recombinant proteins, Ptr4CL3 or Ptr4CL5 was diluted in phosphate buffered saline (PBS) to a final concentration of $200 \mathrm{nM}$ and mixed with individual aliquots of recombinant proteins of PtrHCT1 or PtrHCT6 (200 nM). DSP was then added to the protein mixtures at a 20 -fold molar excess (20:1 DSP:protein). The protein cross-linking was carried out at room temperature for $5 \mathrm{~min}$ and quenched by the addition of $50 \mathrm{mM}$ Tris- $\mathrm{HCl}$ ( $\mathrm{pH}$ 7.5). The DSP-treated and the untreated protein samples were then incubated with sample loading buffer [2\% SDS, $200 \mathrm{mM}$ Tris- $\mathrm{HCl}$ ( $\mathrm{pH} 6.8$ ), 40\% glycerol, and $0.08 \%$ bromophenol blue] and heated to $95^{\circ} \mathrm{C}$ for $5 \mathrm{~min}$, separated by SDS-PAGE, and analyzed by western blotting using Ptr4CL3 and PtrHCT1 antibodies.

\section{DATA AVAILABILITY STATEMENT}

The datasets presented in this study can be found in online repositories. The names of the repository/repositories and accession number(s) can be found in the article/ Supplementary Material.

\section{AUTHOR CONTRIBUTIONS}

C-YL and YS performed the enzyme activity analyses. RS and CY generated the PtrHCT-RNAi transgenic poplar. JL

\section{REFERENCES}

Achnine, L., Blancaflor, E. B., Rasmussen, S., and Dixon, R. A. (2004). Colocalization of L-phenylalanine ammonia-lyase and cinnamate 4hydroxylase for metabolic channeling in phenylpropanoid biosynthesis. Plant Cell 16, 3098-3109. doi: 10.1105/tpc.104.024406

Albersheim, P., Darvill, A., Roberts, K., Sederoff, R., and Staehelin, A. (2010). "The structural polysaccharides of the cell wall and how they are studied," in Plant Cell Walls, (New York, NY: Taylor \& Francis Group), 61-84. doi: 10.1201/9780203833476-6

Anderson, L. E. (1974). Microenvironmental manipulation of the observed Michaelis constant of ribulose diphosphate carboxylase. Plant Physiol. 54, 791-793. doi: 10.1104/pp.54.5.791

Ban, Z., Qin, H., Mitchell, A. J., Liu, B., Zhang, F., Weng, J.-K., et al. (2018). Noncatalytic chalcone isomerase-fold proteins in Humulus lupulus are auxiliary components in prenylated flavonoid biosynthesis. Proc. Natl. Acad. Sci. U.S.A. 115, E5223-E5232.

Barrière, Y., Riboulet, C., Méchin, V., Maltese, S., Pichon, M., Cardinal, A., et al. (2007). Genetics and genomics of lignification in grass cell walls based on maize as model species. Genes Genomes Genomics 1, 133-156.

Barros, J., and Dixon, R. A. (2020). Plant phenylalanine/tyrosine ammonia-lyases. Trends Plant Sci. 25, 66-79. doi: 10.1016/j.tplants.2019.09.011

Barros, J., Escamilla-Trevino, L., Song, L., Rao, X., Serrani-Yarce, J. C., Palacios, M. D., et al. (2019). 4-Coumarate 3-hydroxylase in the lignin biosynthesis pathway is a cytosolic ascorbate peroxidase. Nat. Commun. 10:1994.

Bassard, J.-E., and Halkier, B. A. (2018). How to prove the existence of metabolons? Phytochem. Rev. 17, 211-227. doi: 10.1007/s11101-017-9509-1

Bassard, J. E., Richert, L., Geerinck, J., Renault, H., Duval, F., Ullmann, P., et al. (2012). Protein-protein and protein-membrane associations in the lignin pathway. Plant Cell 24, 4465-4482. doi: 10.1105/tpc.112.102566 performed the enzyme activities of SDX extracts. ST-A and PL performed the RNAseq analysis and protein quantification. JS performed the mechanistic modeling and numerical analysis. $\mathrm{C}-\mathrm{YL}$, YS, and H-CC performed the pull-down and crosslinking experiments. C-YL, YS, JS, CW, DM, Y-CL, RRS, JW and VC analyzed the data. C-YL, YS, JS, and JW wrote the manuscript. JW, RRS, and VC edited the manuscript. C-YL, YS, and JS contributed equally to this work. All the authors contributed to the article and approved the submitted version.

\section{FUNDING}

The National Science Foundation (United States), Plant Genome Research Program grant DBI-0922391 (to VC), the NC State University Jordan Family Endowment, the NC State University Forest Biotechnology Industrial Research Consortium, the Fundamental Research Funds for the Central Universities, China (grants \#2572018CL01 and \#2572020DY24), and the Innovation Project of State Key Laboratory of Tree Genetics and Breeding, Northeast Forestry University, China (grant \#A01) supported this work.

\section{SUPPLEMENTARY MATERIAL}

The Supplementary Material for this article can be found online at: https://www.frontiersin.org/articles/10.3389/fpls.2021. 727932/full\#supplementary-material

Baucher, M., Halpin, C., Petit-Conil, M., and Boerjan, W. (2003). Lignin: genetic engineering and impact on pulping. Crit. Rev. Biochem. Mol. Biol. 38, 305-350. doi: 10.1080/10409230391036757

Biała, W., and Jasiński, M. (2018). The phenylpropanoid case-it is transport that matters. Front. Plant Sci. 9:1610. doi: 10.3389/fpls.2018.01610

Blount, J. W., Korth, K. L., Masoud, S. A., Rasmussen, S., Lamb, C., and Dixon, R. A. (2000). Altering expression of cinnamic acid 4-hydroxylase in transgenic plants provides evidence for a feedback loop at the entry point into the phenylpropanoid pathway. Plant Physiol. 122, 107-116.

Boerjan, W., Ralph, J., and Baucher, M. (2003). Lignin biosynthesis. Annu. Rev. Plant Biol. 54, 519-546.

Bomble, Y. J., Lin, C.-Y., Amore, A., Wei, H., Holwerda, E. K., Ciesielski, P. N., et al. (2017). Lignocellulose deconstruction in the biosphere. Curr. Opin. Chem. Biol. 41, 61-70. doi: 10.1016/j.cbpa.2017.10.013

Brown, S. A., and Neish, A. C. (1955). Shikimic acid as a precursor in lignin biosynthesis. Nature 175, 688-689. doi: 10.1038/175688a0

Burbulis, I. E., and Winkel-Shirley, B. (1999). Interactions among enzymes of the arabidopsis flavonoid biosynthetic pathway. Proc. Natl. Acad. Sci. U.S.A. 96, 12929-12934. doi: 10.1073/pnas.96.22.12929

Campbell, M. M., and Sederoff, R. R. (1996). Variation in lignin content and composition (Mechanisms of control and implications for the genetic improvement of plants). Plant Physiol. 110, 3-13. doi: 10.1104/pp.110.1.3

Carlos del Río, J., Rencoret, J., Gutiérrez, A., Kim, H., and Ralph, J. (2017). Hydroxystilbenes are monomers in palm fruit endocarp lignins. Plant Physiol. 174, 2072-2082. doi: 10.1104/pp.17.00362

Chanoca, A., De Vries, L., and Boerjan, W. (2019). Lignin engineering in forest trees. Front. Plant Sci. 10:912. doi: 10.3389/fpls.2019.00912

Chen, F., Tobimatsu, Y., Havkin-Frenkel, D., Dixon, R. A., and Ralph, J. (2012). A polymer of caffeyl alcohol in plant seeds. Proc. Natl. Acad. Sci. U.S.A. 109, 1772-1777. doi: 10.1073/pnas.1120992109 
Chen, H. C., Li, Q. Z., Shuford, C. M., Liu, J., Muddiman, D. C., Sederoff, R. R., et al. (2011). Membrane protein complexes catalyze both 4-and 3-hydroxylation of cinnamic acid derivatives in monolignol biosynthesis. Proc. Natl. Acad. Sci. U.S.A. 108, 21253-21258. doi: 10.1073/pnas.1116416109

Chen, H. C., Song, J., Wang, J. P., Lin, Y. C., Ducoste, J., Shuford, C. M., et al. (2014). Systems biology of lignin biosynthesis in Populus trichocarpa: heteromeric 4coumaric acid:coenzyme A ligase protein complex formation, regulation, and numerical modeling. Plant Cell 26, 876-893. doi: 10.1105/tpc.113.119685

Chen, H.-C., Song, J., Williams, C. M., Shuford, C. M., Liu, J., Wang, J. P., et al. (2013). Monolignol pathway 4-coumaric acid:coenzyme A ligases in Populus. trichocarpa: novel specificity, metabolic regulation, and simulation of coenzyme a ligation fluxes. Plant Physiol. 161, 1501-1516. doi: 10.1104/pp.112.210971

Crosby, K. C., Pietraszewska-Bogiel, A., Gadella, T. W. J., and Winkel, B. S. J. (2011). Förster resonance energy transfer demonstrates a flavonoid metabolon in living plant cells that displays competitive interactions between enzymes. FEBS Lett. 585, 2193-2198. doi: 10.1016/j.febslet.2011.05.066

Czichi, U., and Kindl, H. (1975). Formation of p-coumaric acid and o-coumaric acid from L-phenylalanine by microsomal membrane fractions from potato: evidence of membrane-bound enzyme complexes. Planta 125, 115-125.

Czichi, U., and Kindl, H. (1977). Phenylalanine ammonia lyase and cinnamic acid hydroxylases as assembled consecutive enzymes on microsomal membranes of cucumber cotyledons: cooperation and subcellular distribution. Planta 134, 133-143. doi: $10.1007 /$ bf00384962

Dastmalchi, M., Bernards, M. A., and Dhaubhadel, S. (2016). Twin anchors of the soybean isoflavonoid metabolon: evidence for tethering of the complex to the endoplasmic reticulum by IFS and C4H. Plant J. 85, 689-706. doi: 10.1111/tpj. 13137

del Río, J. C., Rencoret, J., Gutiérrez, A., Elder, T., Kim, H., and Ralph, J. (2020). Lignin monomers from beyond the canonical monolignol biosynthetic pathway: another brick in the wall. ACS Sustain. Chem. Eng. 8, 4997-5012. doi: 10.1021/acssuschemeng.0c01109

Deng, Y., and Lu, S. (2017). Biosynthesis and regulation of phenylpropanoids in plants. CRC Crit. Rev. Plant Sci. 36, 257-290. doi: 10.1080/07352689.2017. 1402852

Dixon, R. A., Chen, F., Guo, D., and Parvathi, K. (2001). The biosynthesis of monolignols: a "metabolic grid", or independent pathways to guaiacyl and syringyl units? Phytochem. Rev. 57, 1069-1084. doi: 10.1016/s0031-9422(01) 00092-9

Dixon, R. A., Howles, P. A., Lamb, C., He, X.-Z., and Reddy, J. T. (1998). "Prospects for the metabolic engineering of bioactive flavonoids and related phenylpropanoid compounds," in Flavonoids in the Living System, eds J. A. Manthey and B. S. Buslig (Boston, MA: Springer US), 55-66.

Du, J., Zhang, Y., and Zhao, Q. (2018). New components of the lignin biosynthetic metabolon. Trends Plant Sci. 23, 557-559. doi: 10.1016/j.tplants.2018.05.007

Du, X., Gellerstedt, G., and Li, J. (2013). Universal fractionation of lignincarbohydrate complexes (LCCs) from lignocellulosic biomass: an example using spruce wood. Plant J. 74, 328-338. doi: 10.1111/tpj.12124

Du, X., Pérez-Boada, M., Fernández, C., Rencoret, J., Del Río, J. C., JiménezBarbero, J., et al. (2014). Analysis of lignin-carbohydrate and lignin-lignin linkages after hydrolase treatment of xylan-lignin, glucomannan-lignin and glucan-lignin complexes from spruce wood. Planta 239, 1079-1090.

Eckardt, N. A. (2002). Probing the mysteries of lignin biosynthesis: the crystal structure of caffeic acid/5-hydroxyferulic acid 3/5-O-methyltransferase provides new insights. Plant Cell 14, 1185-1189. doi: 10.1105/tpc.140610

Ehlert, A., Weltmeier, F., Wang, X., Mayer, C. S., Smeekens, S., Vicente-Carbajosa, J., et al. (2006). Two-hybrid protein-protein interaction analysis in Arabidopsis protoplasts: establishment of a heterodimerization map of group $\mathrm{C}$ and group $\mathrm{S}$ bZIP transcription factors. Plant J. 46, 890-900. doi: 10.1111/j.1365-313x.2006. 02731.x

Ehlting, J., Büttner, D., Wang, Q., Douglas, C. J., Somssich, I. E., and Kombrink, E. (1999). Three 4-coumarate:coenzyme A ligases in Arabidopsis thaliana represent two evolutionarily divergent classes in angiosperms. Plant J. 19, 9-20. doi: 10.1046/j.1365-313x.1999.00491.x

Faraji, M., Fonseca, L. L., Escamilla-Treviño, L., Barros-Rios, J., Engle, N., Yang, Z. K., et al. (2018). Mathematical models of lignin biosynthesis. Biotechnol. Biofuels 11:34.

Faraji, M., Fonseca, L. L., Escamilla-Treviño, L., Dixon, R. A., and Voit, E. O. (2015). Computational inference of the structure and regulation of the lignin pathway in Panicum virgatum. Biotechnol. Biofuels 8:151.
Fritsch, H., and Grisebach, H. (1975). Biosynthesis of cyanidin in cell cultures of Haplopappus gracilis. Phytochemistry 14, 2437-2442. doi: 10.1016/00319422(75)80360-8

Fujino, N., Tenma, N., Waki, T., Ito, K., Komatsuzaki, Y., Sugiyama, K., et al. (2018). Physical interactions among flavonoid enzymes in snapdragon and torenia reveal the diversity in the flavonoid metabolon organization of different plant species. Plant J. 94, 372-392. doi: 10.1111/tpj.13864

Gaertner, F. H., Ericson, M. C., and Demoss, J. A. (1970). Catalytic facilitation in vitro by two multienyzme complexes from Neurospora crassa. J. Biol. Chem. 245, 595-600. doi: 10.1016/s0021-9258(18)63373-3

Giles, N. H., Case, M. E., Partridge, C. W., and Ahmed, S. I. (1967). A gene cluster in Nuerospora crassa coding for an aggregate of five aromatic synthetic enzymes. Proc. Natl. Acad. Sci. U.S.A. 58, 1453-1460. doi: 10.1073/pnas.58.4.1453

Gillet, S., Aguedo, M., Petitjean, L., Morais, A. R. C., Da Costa Lopes, A. M., Łukasik, R. M., et al. (2017). Lignin transformations for high value applications: towards targeted modifications using green chemistry. Green Chem. 19, 42004233. doi: $10.1039 / \mathrm{c7gc01479a}$

Ginsburg, A., and Stadtman, E. R. (1970). Multienzyme systems. Annu. Rev. Biochem. 39, 429-472.

Gou, M., Ran, X., Martin, D. W., and Liu, C. J. (2018). The scaffold proteins of lignin biosynthetic cytochrome P450 enzymes. Nat. Plants 4, 299-310. doi: 10.1038/s41477-018-0142-9

Gross, G. G. (1979). "Recent advances in the chemistry and biochemistry of lignin," in Biochemistry of Plant Phenolics, eds T. Swain, J. B. Harbone, and C. F. Van Sumere (Boston, MA: Springer US), 177-220. doi: 10.1007/978-1-4684-33722_6

Guo, D.-M., Ran, J.-H., and Wang, X.-Q. (2010). Evolution of the cinnamyl/sinapyl alcohol dehydrogenase (CAD/SAD) gene family: the emergence of real lignin is associated with the origin of bona fide CAD. J. Mol. Evol. 71, 202-218. doi: 10.1007/s00239-010-9378-3

Gupta, V. K., Potumarthi, R., O’donovan, A., Kubicek, C. P., Sharma, G. D., and Tuohy, M. G. (2014). "Bioenergy research: an overview on technological developments and bioresources," in Bioenergy Research: Advances and Applications, eds V. K. Gupta, M. G. Tuohy, C. P. Kubicek, J. Saddler, and F. $\mathrm{Xu}$ (Amsterdam: Elsevier), 23-47.

Hamberger, B., Ellis, M., Friedmann, M., De Azevedo Souza, C., Barbazuk, B., and Douglas, C. J. (2007). Genome-wide analyses of phenylpropanoid-related genes in Populus trichocarpa, Arabidopsis thaliana, and Oryza sativa: the Populus lignin toolbox and conservation and diversification of angiosperm gene families. Can. J. Bot. 85, 1182-1201. doi: 10.1139/b07-098

He, X.-Z., and Dixon, R. A. (2000). Genetic manipulation of isoflavone 7O-methyltransferase enhances biosynthesis of 4'-O-methylated isoflavonoid phytoalexins and disease resistance in alfalfa. Plant Cell 12, 1689-1702. doi: $10.2307 / 3871183$

Higuchi, T. (1985). Biosynthesis and Biodegradation of Wood Components. Orlando, FL: Academic Press.

Higuchi, T. (1990). Lignin biochemistry: biosynthesis and biodegradation. Wood Sci. Technol. 24, 23-63.

Higuchi, T. (1997). Biochemistry and Molecular Biology of Wood. Berlin: SpringerVerlag.

Higuchi, T. (2003). Pathways for monolignol biosynthesis via metabolic grids: coniferyl aldehyde 5-hydroxylase, a possible key enzyme in angiosperm syringyl lignin biosynthesis. Proc. Jpn. Acad. Ser. B Phys. Biol. Sci. 79, 227-236. doi: $10.2183 /$ pjab.79b. 227

Himmel, M. E. (2009). Biomass Recalcitrance: Deconstructing the Plant Cell Wall for Bioenergy. Hoboken, NJ: Wiley-Blackwell.

Hrazdina, G. (1992). "Compartmentation in aromatic metabolism," in Phenolic Metabolism in Plants, eds H. A. Stafford and R. K. Ibrahim (Boston, MA: Springer US), 1-23. doi: 10.1007/978-1-4615-3430-3_1

Hrazdina, G., and Jensen, R. A. (1992). Spatial organization of enzymes in plant metabolic pathways. Annu. Rev. Plant Physiol. Plant Mol. Biol. 43, 241-267. doi: 10.1146/annurev.pp.43.060192.001325

Hrazdina, G., and Wagner, G. J. (1985a). Compartmentation of plant phenolic compounds; site of synthesis and accumulation. Annu. Proc. Phytochem. Soc. Europe 25, 119-133.

Hrazdina, G., and Wagner, G. J. (1985b). Metabolic pathways as enzyme complexes: evidence for the synthesis of phenylpropanoids and flavonoids on membrane associated enzyme complexes. Arch. Biochem. Biophys. 237, 88-100. doi: 10. 1016/0003-9861(85)90257-7 
Hrazdina, G., Wagner, G. J., and Siegelman, H. W. (1978). Subcellular localization of enzymes of anthocyanin biosynthesis in protoplasts. Phytochemistry 17, 53-56. doi: 10.1016/s0031-9422(00)89679-x

Hrazdina, G., Zobel, A. M., and Hoch, H. C. (1987). Biochemical, immunological, and immunocytochemical evidence for the association of chalcone synthase with endoplasmic reticulum membranes. Proc. Natl. Acad. Sci. U.S.A. 84, 8966-8970. doi: 10.1073/pnas.84.24.8966

Hu, W.-J., Harding, S. A., Lung, J., Popko, J. L., Ralph, J., Stokke, D. D., et al. (1999). Repression of lignin biosynthesis promotes cellulose accumulation and growth in transgenic trees. Nat. Biotechnol. 17, 808-812. doi: 10.1038/11758

Hu, W.-J., Kawaoka, A., Tsai, C.-J., Lung, J., Osakabe, K., Ebinuma, H., et al. (1998). Compartmentalized expression of two structurally and functionally distinct 4coumarate:CoA ligase genes in aspen (Populus tremuloides). Proc. Natl. Acad. Sci. U.S.A. 95, 5407-5412. doi: 10.1073/pnas.95.9.5407

Jacques, D., Opie, C. T., Porter, L. J., and Haslam, E. (1977). Plant proanthocyanidins. Part 4. Biosynthesis of procyanidins and observations on the metabolism of cyanidin in plants. J. Chem. Soc. Perkin Trans. 1, 1637-1643. doi: 10.1039/p19770001637

Jin, Z., Katsumata, K. S., Lam, T. B. T., and Iiyama, K. (2006). Covalent linkages between cellulose and lignin in cell walls of coniferous and nonconiferous woods. Biopolymers 83, 103-110. doi: 10.1002/bip.20533

Jorgensen, K., Rasmussen, A. V., Morant, M., Nielsen, A. H., Bjarnholt, N., Zagrobelny, M., et al. (2005). Metabolon formation and metabolic channeling in the biosynthesis of plant natural products. Curr. Opin. Plant Biol. 8, 280-291. doi: 10.1016/j.pbi.2005.03.014

Jung, H. G. (1989). Forage lignins and their effects on fiber digestibility. Agron. J. 81, 33-38. doi: 10.2134/agronj1989.00021962008100010006x

Kim, H., Li, Q., Karlen, S. D., Smith, R. A., Shi, R., Liu, J., et al. (2020). Monolignol benzoates incorporate into the lignin of transgenic Populus trichocarpa depleted in $\mathrm{C} 3 \mathrm{H}$ and $\mathrm{C} 4 \mathrm{H}$. ACS Sustain. Chem. Eng. 8, 3644-3654. doi: 10.1021/ acssuschemeng. 9 b06389

Lan, W., Lu, F., Regner, M., Zhu, Y., Rencoret, J., Ralph, S. A., et al. (2015). Tricin, a flavonoid monomer in monocot lignification. Plant Physiol. 167, 1284-1295. doi: 10.1104/pp.114.253757

Laursen, T., Borch, J., Knudsen, C., Bavishi, K., Torta, F., Martens, H. J., et al. (2016). Characterization of a dynamic metabolon producing the defense compound dhurrin in sorghum. Science 354, 890-893. doi: 10.1126/science. aag2347

Laursen, T., Møller, B. L., and Bassard, J.-E. (2015). Plasticity of specialized metabolism as mediated by dynamic metabolons. Trends Plant Sci. 20, 20-32. doi: 10.1016/j.tplants.2014.11.002

Lee, Y., Escamilla-Treviño, L., Dixon, R. A., and Voit, E. O. (2012). Functional analysis of metabolic channeling and regulation in lignin biosynthesis: a computational approach. PLoS Comput. Biol. 8:e1002769. doi: 10.1371/journal. pcbi.1002769

Lee, Y., and Voit, E. O. (2010). Mathematical modeling of monolignol biosynthesis in Populus xylem. Math. Biosci. 228, 78-89. doi: 10.1016/j.mbs.2010.08.009

Li, J., Ou-Lee, T. M., Raba, R., Amundson, R. G., and Last, R. L. (1993). Arabidopsis flavonoid mutants are hypersensitive to UV-B irradiation. Plant cell 5, 171-179. doi: $10.2307 / 3869583$

Liang, L., Xiao-Bing, W., Lei, Y., and Ling-Yi, K. (2010). Quantitative evaluation of 4-coumarate: CoA ligase (4CL) activity and correlated chemical constituents in four plant materials by chromatographic analysis. Chin. J. Nat. Med. 8, 274-279. doi: 10.3724/sp.j.1009.2010.00274

Lin, C.-Y., and Eudes, A. (2020). Strategies for the production of biochemicals in bioenergy crops. Biotechnol. Biofuels 13:71.

Lin, C.-Y., Li, Q., Tunlaya-Anukit, S., Shi, R., Sun, Y.-H., Wang, J. P., et al. (2016). A cell wall-bound anionic peroxidase, PtrPO21, is involved in lignin polymerization in Populus trichocarpa. Tree Genet. Genomes 12:22.

Lin, C.-Y., Wang, J. P., Li, Q., Chen, H.-C., Liu, J., Loziuk, P., et al. (2015). 4-Coumaroyl and caffeoyl shikimic acids inhibit 4-coumaric acid:Coenzyme A ligases and modulate metabolic flux for 3-hydroxylation in monolignol biosynthesis of Populus trichocarpa. Mol. Plant 8, 176-187. doi: 10.1016/j.molp. 2014.12.003

Lin, Y. C., Li, W., Chen, H., Li, Q., Sun, Y. H., Shi, R., et al. (2014). A simple improved-throughput xylem protoplast system for studying wood formation. Nat. Protoc. 9, 2194-2205. doi: 10.1038/nprot.2014.147
Liu, C.-J. (2012). Deciphering the enigma of lignification: precursor transport, oxidation, and the topochemistry of lignin assembly. Mol. Plant 5, 304-317. doi: $10.1093 / \mathrm{mp} / \mathrm{ssr} 121$

Liu, C.-J., Blount, J. W., Steele, C. L., and Dixon, R. A. (2002). Bottlenecks for metabolic engineering of isoflavone glycoconjugates in Arabidopsis. Proc. Natl. Acad. Sci. U.S.A. 99, 14578-14583. doi: 10.1073/pnas.212522099

Liu, C.-J., and Dixon, R. A. (2001). Elicitor-induced association of isoflavone $\mathrm{O}$-methyltransferase with endomembranes prevents the formation and 7-Omethylation of daidzein during isoflavonoid phytoalexin biosynthesis. Plant Cell 13, 2643-2658. doi: 10.1105/tpc.13.12.2643

Liu, J., Shi, R., Li, Q., Sederoff, R. R., and Chiang, V. L. (2012). A standard reaction condition and a single HPLC separation system are sufficient for estimation of monolignol biosynthetic pathway enzyme activities. Planta 236, 879-885. doi: 10.1007/s00425-012-1688-9

Lomant, A. J., and Fairbanks, G. (1976). Chemical probes of extended biological structures: Synthesis and properties of the cleavable protein cross-linking reagent $\left[{ }^{35}\right.$ S $]$ dithiobis(succinimidyl propionate). J. Mol. Biol. 104, 243-261. doi: 10.1016/0022-2836(76)90011-5

Loscher, R., and Heide, L. (1994). Biosynthesis of p-hydroxybenzoate from p-coumarate and p-coumaroyl-Coenzyme $\mathrm{A}$ in cell-free extracts of Lithospermum erythrorhizon cell cultures. Plant Physiol. 106, 271-279. doi: 10.1104/pp.106.1.271

Lu, S., Li, Q., Wei, H., Chang, M.-J., Tunlaya-Anukit, S., Kim, H., et al. (2013). Ptr-miR397a is a negative regulator of laccase genes affecting lignin content in Populus trichocarpa. Proc. Natl. Acad. Sci. U.S.A. 110, 10848-10853. doi: 10.1073/pnas.1308936110

Luckner, M. (1984). "Cellular compartmentalization and channeling," in Secondary Metabolism in Microorganisms, Plants and Animals (Berlin: Springer Nature Group), 31-43. doi: 10.1007/978-3-662-02384-6_3

MacKay, J. J., O’Malley, D. M., Presnell, T., Booker, F. L., Campbell, M. M., Whetten, R. W., et al. (1997). Inheritance, gene expression, and lignin characterization in a mutant pine deficient in cinnamyl alcohol dehydrogenase. Proc. Natl. Acad. Sci. U.S.A. 94, 8255-8260. doi: 10.1073/pnas.94.15.8255

Mameda, R., Waki, T., Kawai, Y., Takahashi, S., and Nakayama, T. (2018). Involvement of chalcone reductase in the soybean isoflavone metabolon: identification of GmCHR5, which interacts with 2-hydroxyisoflavanone synthase. Plant J. 96, 56-74. doi: 10.1111/tpj.14014

Masters, C. J. (1977). "Metabolic control and the microenvironment," in Current Topics in Cellular Regulation, eds B. L. Horecker and E. R. Stadtman (New York, NY: Academic Press), 75-105. doi: 10.1016/b978-0-12-152812-6.50009-3

Meselson, M., Stahl, F. W., and Vinograd, J. (1957). Equilibrium sedimentation of macromolecules in density gradients. Proc. Natl. Acad. Sci. U.S.A. 43, 581-588. doi: 10.1073/pnas.43.7.581

Miedes, E., Vanholme, R., Boerjan, W., and Molina, A. (2014). The role of the secondary cell wall in plant resistance to pathogens. Front. Plant Sci. 5:358. doi: $10.3389 /$ fpls.2014.00358

Miles, E. W. (2001). Tryptophan synthase: a multienzyme complex with an intramolecular tunnel. Chem. Rec. 1, 140-151. doi: 10.1002/tcr.4

Mork-Jansson, A. E., and Eichacker, L. A. (2019). A strategy to characterize chlorophyll protein interaction in LIL3. Plant Methods 15:1.

Mottiar, Y., Vanholme, R., Boerjan, W., Ralph, J., and Mansfield, S. D. (2016). Designer lignins: harnessing the plasticity of lignification. Curr. Opin. Biotechnol. 37, 190-200. doi: 10.1016/j.copbio.2015.10.009

Mucha, S., Heinzlmeir, S., Kriechbaumer, V., Strickland, B., Kirchhelle, C., Choudhary, M., et al. (2019). The formation of a camalexin biosynthetic metabolon. Plant Cell 31, 2697-2710.

Nakayama, T., Takahashi, S., and Waki, T. (2019). Formation of flavonoid metabolons: functional significance of protein-protein interactions and impact on flavonoid chemodiversity. Front. Plant Sci. 10:821. doi: 10.3389/fpls.2019. 00821

Nanayakkara, B., Manley-Harris, M., Suckling, I. D., and Donaldson, L. A. (2009). Quantitative chemical indicators to assess the gradation of compression wood. Holzforschung 63:431.

Nester, E. W., Lorence, J. H., and Nasser, D. S. (1967). An enzyme aggregate involved in the biosynthesis of aromatic amino acids in Bacillus subtilis. Its possible function in feedback regulation. Biochemistry 6, 1553-1563.

Novaes, E., Kirst, M., Chiang, V., Winter-Sederoff, H., and Sederoff, R. (2010). Lignin and biomass: a negative correlation for wood formation and lignin 
content in trees. Plant Physiol. 154, 555-561. doi: 10.1104/pp.110.16 1281

Obata, T. (2019). Metabolons in plant primary and secondary metabolism. Phytochem. Rev. 18, 1483-1507. doi: 10.1007/s11101-019-09619-x

Ovádi, J. (1991). Physiological significance of metabolic channelling. J. Theor. Biol. 152, 1-22. doi: 10.1016/s0022-5193(05)80500-4

Ovádi, J., and Sreret, P. A. (1999). "Macromolecular compartmentation and channeling," in International Review of Cytology, eds H. Walter, D. E. Brooks, and P. A. Srere (New York, NY: Academic Press), 255-280. doi: 10.1016/s00747696(08)60529-X

Panicot, M., Minguet, E. G., Ferrando, A., Alcázar, R., Blázquez, M. A., Carbonell, J., et al. (2002). A polyamine metabolon involving aminopropyl transferase complexes in Arabidopsis. Plant Cell 14, 2539-2551. doi: 10.1105/tpc.004077

Pauly, M., and Keegstra, K. (2008). Cell-wall carbohydrates and their modification as a resource for biofuels. Plant J. 54, 559-568.

Payen, A. (1839). Recherches sur la matière incrustante des bois. Acad. Sci. Paris Compt. Rend. 8, 169-170.

Peña-Castro, J. M., Del Moral, S., Núñez-López, L., Barrera-Figueroa, B. E., and Amaya-Delgado, L. (2017). Biotechnological strategies to improve plant biomass quality for bioethanol production. Biomed. Res. Int. 2017:7824076.

Pettersson, G. (1991). No convincing evidence is available for metabolite channelling between enzymes forming dynamic complexes. J. Theor. Biol. 152, 65-69. doi: 10.1016/s0022-5193(05)80512-0

Poovaiah, C. R., Nageswara-Rao, M., Soneji, J. R., Baxter, H. L., and Stewart, C. N. Jr. (2014). Altered lignin biosynthesis using biotechnology to improve lignocellulosic biofuel feedstocks. Plant Biotechnol. J. 12, 1163-1173. doi: 10. $1111 /$ pbi. 12225

Radloff, R., Bauer, W., and Vinograd, J. (1967). A dye-buoyant-density method for the detection and isolation of closed circular duplex DNA: the closed circular DNA in HeLa cells. Proc. Natl. Acad. Sci. U.S.A. 57, 1514-1521. doi: 10.1073/ pnas.57.5.1514

Raes, J., Rohde, A., Christensen, J. H., Van De Peer, Y., and Boerjan, W. (2003). Genome-wide characterization of the lignification toolbox in Arabidopsis. Plant Physiol. 133, 1051-1071. doi: 10.1104/pp.103.026484

Ragauskas, A. J., Beckham, G. T., Biddy, M. J., Chandra, R., Chen, F., Davis, M. F., et al. (2014). Lignin valorization: improving lignin processing in the biorefinery. Science 344:1246843. doi: 10.1126/science. 1246843

Ralph, J. (2010). Hydroxycinnamates in lignification. Phytochem. Rev. 9, 65-83. doi: 10.1007/s11101-009-9141-9

Ralph, J., Lapierre, C., and Boerjan, W. (2019). Lignin structure and its engineering. Curr. Opin. Biotechnol. 56, 240-249. doi: 10.1016/j.copbio.2019.02.019

Ralph, J., Lundquist, K., Brunow, G., Lu, F., Kim, H., Schatz, P. F., et al. (2004). Lignins: natural polymers from oxidative coupling of 4-hydroxyphenylpropanoids. Phytochem. Rev. 3, 29-60. doi: 10.1023/b:phyt.0000047809.65444. a4

Ralph, J., Mackay, J. J., Hatfield, R. D., O'malley, D. M., Whetten, R. W., and Sederoff, R. R. (1997). Abnormal lignin in a loblolly pine mutant. Science 277, 235-239. doi: 10.1126/science.277.5323.235

Ralston, L., and Yu, O. (2006). Metabolons involving plant cytochrome P450s. Phytochem. Rev. 5, 459-472. doi: 10.1007/s11101-006-9014-4

Rasmussen, S., and Dixon, R. A. (1999). Transgene-mediated and elicitorinduced perturbation of metabolic channeling at the entry point into the phenylpropanoid pathway. Plant Cell 11, 1537-1552. doi: 10.2307/3870981

Ro, D.-K., and Douglas, C. J. (2004). Reconstitution of the entry point of plant phenylpropanoid metabolism in yeast (Saccharomyces cerevisiae): implications for control of metabolic flux into the phenylpropanoid pathway. J. Biol. Chem. 279, 2600-2607. doi: 10.1074/jbc.m309951200

Rowell, R. M., Pettersen, R., Han, J. S., Rowell, J. S., and Tshabalala, M. A. (2005). "Cell wall chemistry," in Handbook of Wood Chemistry and Wood Composites (Boca Raton, FL: Taylor \& Francis Group), 33-72.

Saleme, M. D. L. S., Cesarino, I., Vargas, L., Kim, H., Vanholme, R., Goeminne, G., et al. (2017). Silencing caffeoyl shikimate esterase affects lignification and improves saccharification in poplar. Plant Physiol. 175, 1040-1057. doi: 10. 1104/pp.17.00920

Sambrook, J., Fritsch, E. F., and Maniatis, T. (1982). Molecular Cloning : A Laboratory Manual. New York, NY: CSH.

Sambrook, J., and Russell, D. W. (2006). Preparation of plasmid DNA by alkaline lysis with sds: maxipreparation. CSH Protoc. 2006:pdb.prot4090. doi: 10.1101/ pdb.prot 4090
Sarkanen, K. V., and Ludwig, C. H. (1971). Lignins: Occurrence, Formation, Structure and Reactions. New York, NY: Wiley-Interscience, 228-230.

Sarkar, P., Bosneaga, E., and Auer, M. (2009). Plant cell walls throughout evolution: towards a molecular understanding of their design principles. J. Exp. Bot. 60, 3615-3635. doi: 10.1093/jxb/erp245

Schmitt, D. L., and An, S. (2017). Spatial organization of metabolic enzyme complexes in cells. Biochemistry 56, 3184-3196. doi: 10.1021/acs.biochem. $7 \mathrm{~b} 00249$

Schwarz, G. (1978). Estimating the dimension of a model. Ann. Statist. 6, 461-464.

Sederoff, R. R., Mackay, J. J., Ralph, J., and Hatfield, R. D. (1999). Unexpected variation in lignin. Curr. Opin. Plant Biol. 2, 145-152. doi: 10.1016/s13695266(99)80029-6

Shen, H., Mazarei, M., Hisano, H., Escamilla-Trevino, L., Fu, C., Pu, Y., et al. (2013). A genomics approach to deciphering lignin biosynthesis in switchgrass. Plant Cell 25, 4342-4361. doi: 10.1105/tpc.113.118828

Shi, R., Sun, Y. H., Li, Q., Heber, S., Sederoff, R., and Chiang, V. L. (2010). Towards a systems approach for lignin biosynthesis in Populus trichocarpa: transcript abundance and specificity of the monolignol biosynthetic genes. Plant Cell Physiol. 51, 144-163. doi: 10.1093/pcp/pcp175

Shih, C. H., Chu, H., Tang, L. K., Sakamoto, W., Maekawa, M., Chu, I. K., et al. (2008). Functional characterization of key structural genes in rice flavonoid biosynthesis. Planta 228, 1043-1054. doi: 10.1007/s00425-008-0806-1

Shuford, C. M., Li, Q., Sun, Y.-H., Chen, H.-C., Wang, J., Shi, R., et al. (2012). Comprehensive quantification of monolignol-pathway enzymes in Populus trichocarpa by protein cleavage isotope dilution mass spectrometry. J. Proteome Res. 11, 3390-3404. doi: 10.1021/pr300205a

Song, J. A. (2014). Mechanistic Model Development for Multi-Enzymatic Reactions in Lignin Biosynthesis. Ph.D. dissertation. Raleigh, NC: North Carolina State University.

Srere, P. A. (1985). The metabolon. Trends Biochem. Sci. 10, 109-110.

Stafford, H. A. (1974a). "Possible multienzyme complexes regulating the formation of C6-C3 phenolic compounds and lignins in higher plants," in Recent Advances in Phytochemistry, eds V. C. Runeckles and E. E. Conn (Amsterdam: Elsevier), 53-79. doi: 10.1016/b978-0-12-612408-8.50009-3

Stafford, H. A. (1974b). The metabolism of aromatic compounds. Annu. Rev. Plant Physiol. 25, 459-486.

Stafford, H. A. (1981). "Compartmentation in natural product biosynthesis by multienzyme complexes," in Secondary Plant Products, ed. E. E. Conn (San Diego, CA: Academic Press), 117-137. doi: 10.1016/b978-0-12-675407-0. 50011-7

Studer, M. H., Demartini, J. D., Davis, M. F., Sykes, R. W., Davison, B., Keller, M., et al. (2011). Lignin content in natural Populus variants affects sugar release. Proc. Natl. Acad. Sci. U.S.A. 108, 6300-6305. doi: 10.1073/pnas.1009252108

Sun, Y., and Cheng, J. (2002). Hydrolysis of lignocellulosic materials for ethanol production: a review. Bioresour. Technol. 83, 1-11. doi: 10.1016/s09608524(01)00212-7

Sweetlove, L. J., and Fernie, A. R. (2013). The spatial organization of metabolism within the plant cell. Annu. Rev. Plant Biol. 64, 723-746. doi: 10.1146/annurevarplant-050312-120233

Tang, X., and Bruce, J. E. (2010). A new cross-linking strategy: protein interaction reporter (PIR) technology for protein-protein interaction studies. Mol. Biosyst. 6, 939-947. doi: 10.1039/b920876c

Tarasov, D., Leitch, M., and Fatehi, P. (2018). Lignin-carbohydrate complexes: properties, applications, analyses, and methods of extraction: a review. Biotechnol. Biofuels 11:269.

Tartof, K. (1987). Improved media for growing plasmid and cosmid clones. Bethesda Res. Lab. Focus 9:12.

Tzin, V., and Galili, G. (2010). New insights into the shikimate and aromatic amino acids biosynthesis pathways in plants. Mol. Plant 3, 956-972. doi: 10.1093/mp/ ssq048

Upton, B. M., and Kasko, A. M. (2016). Strategies for the conversion of lignin to high-value polymeric materials: review and perspective. Chem. Rev. 116, 2275-2306. doi: 10.1021/acs.chemrev.5b00345

Vanholme, R., Demedts, B., Morreel, K., Ralph, J., and Boerjan, W. (2010). Lignin biosynthesis and structure. Plant Physiol. 153, 895-905.

Vanholme, R., Morreel, K., Darrah, C., Oyarce, P., Grabber, J. H., Ralph, J., et al. (2012). Metabolic engineering of novel lignin in biomass crops. New Phytol. 196, 978-1000. doi: 10.1111/j.1469-8137.2012.04337.x 
Vanholme, R., Morreel, K., Ralph, J., and Boerjan, W. (2008). Lignin engineering. Curr. Opin. Plant Biol. 11, 278-285.

Vogt, T. (2010). Phenylpropanoid biosynthesis. Mol. Plant 3, 2-20. doi: 10.1093/ $\mathrm{mp} / \mathrm{ssp} 106$

Wagner, G. J., and Hrazdina, G. (1984). Endoplasmic reticulum as a site of phenylpropanoid and flavonoid metabolism in hippeastrum. Plant Physiol. 74, 901-906. doi: 10.1104/pp.74.4.901

Waki, T., Yoo, D., Fujino, N., Mameda, R., Denessiouk, K., Yamashita, S., et al. (2016). Identification of protein-protein interactions of isoflavonoid biosynthetic enzymes with 2-hydroxyisoflavanone synthase in soybean (Glycine max (L.) Merr.). Biochem. Biophys. Res. Commun. 469, 546-551. doi: 10.1016/ j.bbrc.2015.12.038

Walker, A. M., Hayes, R. P., Youn, B., Vermerris, W., Sattler, S. E., and Kang, C. (2013). Elucidation of the structure and reaction mechanism of sorghum hydroxycinnamoyltransferase and its structural relationship to other coenzyme a-dependent transferases and synthases. Plant Physiol. 162, 640-651. doi: 10. 1104/pp.113.217836

Wang, J. P., Matthews, M. L., Williams, C. M., Shi, R., Yang, C., Tunlaya-Anukit, S., et al. (2018). Improving wood properties for wood utilization through multi-omics integration in lignin biosynthesis. Nat. Commun. 9:1579.

Wang, J. P., Naik, P. P., Chen, H.-C., Shi, R., Lin, C.-Y., Liu, J., et al. (2014). Complete proteomic-based enzyme reaction and inhibition kinetics reveal how monolignol biosynthetic enzyme families affect metabolic flux and lignin in Populus trichocarpa. Plant Cell 26, 894-914. doi: 10.1105/tpc.113.120881

Wang, J. P., Shuford, C. M., Li, Q. Z., Song, J. N., Lin, Y. C., Sun, Y. H., et al. (2012). Functional redundancy of the two 5-hydroxylases in monolignol biosynthesis of Populus trichocarpa: LC-MS/MS based protein quantification and metabolic flux analysis. Planta 236, 795-808. doi: 10.1007/s00425-012-1663-5

Weid, M., Ziegler, J., and Kutchan, T. M. (2004). The roles of latex and the vascular bundle in morphine biosynthesis in the opium poppy, Papaver somniferum. Proc. Natl. Acad. Sci. U.S.A. 101, 13957-13962. doi: 10.1073/pnas.0405704101

Welker, C. M., Balasubramanian, V. K., Petti, C., Rai, K. M., Debolt, S., and Mendu, V. (2015). Engineering plant biomass lignin content and composition for biofuels and bioproducts. Energies 8, 7654-7676. doi: 10.3390/en8087654

Weng, J.-K., and Chapple, C. (2010). The origin and evolution of lignin biosynthesis. New Phytol. 187, 273-285. doi: 10.1111/j.1469-8137.2010.03327.x

Weng, J.-K., Li, X., Bonawitz, N. D., and Chapple, C. (2008). Emerging strategies of lignin engineering and degradation for cellulosic biofuel production. Curr. Opin. Biotechnol. 19, 166-172. doi: 10.1016/j.copbio.2008.02.014

Whetten, R., and Sederoff, R. (1995). Lignin biosynthesis. Plant Cell 7, 1001-1013.

Wilkerson, C. G., Mansfield, S. D., Lu, F., Withers, S., Park, J.-Y., Karlen, S. D., et al. (2014). Monolignol ferulate transferase introduces chemically labile linkages into the lignin backbone. Science 344, 90-93. doi: 10.1126/science.1250161

Williams, T. C. R., Sweetlove, L. J., and Ratcliffe, R. G. (2011). Capturing metabolite channeling in metabolic flux phenotypes. Plant Physiol. 157, 981-984.
Winkel, B. S. J. (2004). Metabolic channeling in plants. Annu. Rev. Plant Biol. 55, 85-107. doi: 10.1146/annurev.arplant.55.031903.141714

Winkel, B. S. J. (2009). "Metabolite channeling and multi-enzyme complexes," in Plant-Derived Natural Products: Synthesis, Function, and Application, eds A. E. Osbourn and V. Lanzotti (New York, NY: Springer US), 195-208. doi: 10.1007/978-0-387-85498-4_9

Winkel-Shirley, B. (1999). Evidence for enzyme complexes in the phenylpropanoid and flavonoid pathways. Physiol. Plant 107, 142-149. doi: 10.1034/j.1399-3054. 1999.100119.x

Winkel-Shirley, B. (2001). Flavonoid biosynthesis. A colorful model for genetics, biochemistry, cell biology, and biotechnology. Plant Physiol. 126, 485-493. doi: 10.1104/pp.126.2.485

Yan, X., Liu, J., Kim, H., Liu, B., Huang, X., Yang, Z., et al. (2019). CAD1 and CCR2 protein complex formation in monolignol biosynthesis in Populus trichocarpa. New Phytol. 222, 244-260. doi: 10.1111/nph.15505

Yeh, T.-F., Braun, J. L., Goldfarb, B., Chang, H.-M., and Kadla, J. F. (2006). Morphological and chemical variations between juvenile wood, mature wood, and compression wood of loblolly pine (Pinus taeda L.). Holzforschung 60:1. doi: 10.1515/hf.2006.001

Zhang, X., and Liu, C.-J. (2015). Multifaceted regulations of gateway enzyme phenylalanine ammonia-lyase in the biosynthesis of phenylpropanoids. Mol. Plant 8, 17-27. doi: 10.1016/j.molp.2014.11.001

Zhang, Y., and Fernie, A. R. (2020). Metabolons, enzyme-enzyme assemblies that mediate substrate channeling, and their roles in plant metabolism. Plant Commun. 2:100081. doi: 10.1016/j.xplc.2020.100081

Zhao, Q., Wang, H., Yin, Y., Xu, Y., Chen, F., and Dixon, R. A. (2010). Syringyl lignin biosynthesis is directly regulated by a secondary cell wall master switch. Proc. Natl. Acad. Sci. U.S.A. 107, 14496-14501. doi: 10.1073/pnas.1009170107

Conflict of Interest: The authors declare that the research was conducted in the absence of any commercial or financial relationships that could be construed as a potential conflict of interest.

Publisher's Note: All claims expressed in this article are solely those of the authors and do not necessarily represent those of their affiliated organizations, or those of the publisher, the editors and the reviewers. Any product that may be evaluated in this article, or claim that may be made by its manufacturer, is not guaranteed or endorsed by the publisher.

Copyright $\odot 2021$ Lin, Sun, Song, Chen, Shi, Yang, Liu, Tunlaya-Anukit, Liu, Loziuk, Williams, Muddiman, Lin, Sederoff, Wang and Chiang. This is an open-access article distributed under the terms of the Creative Commons Attribution License (CC BY). The use, distribution or reproduction in other forums is permitted, provided the original author(s) and the copyright owner(s) are credited and that the original publication in this journal is cited, in accordance with accepted academic practice. No use, distribution or reproduction is permitted which does not comply with these terms. 